\title{
Prompt photons in photoproduction at HERA
}

\section{The H1 Collaboration}

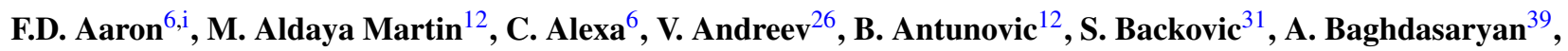
E. Barrelet ${ }^{30}$, W. Bartel ${ }^{12}$, K. Begzsuren ${ }^{36}$, A. Belousov ${ }^{26}$, J.C. Bizot ${ }^{28}$, V. Boudry ${ }^{29}$, I. Bozovic-Jelisavcic ${ }^{2}$, J. Bracinik ${ }^{3}$, G. Brandt ${ }^{12}$, M. Brinkmann ${ }^{13}$, V. Brisson ${ }^{28}$, D. Bruncko ${ }^{17}$, A. Bunyatyan ${ }^{14,39}$, G. Buschhorn ${ }^{27}$, L. Bystritskaya ${ }^{25}$, A.J. Campbell ${ }^{12}$, K.B. Cantun Avila ${ }^{23}$, K. Cerny ${ }^{33}$, V. Cerny ${ }^{17, g}$, V. Chekelian ${ }^{27}$, A. Cholewa ${ }^{12}$, J.G. Contreras ${ }^{23}$, J.A. Coughlan ${ }^{7}$, G. Cozzika ${ }^{11}$, J. Cvach ${ }^{32}$, J.B. Dainton ${ }^{19}$, K. Daum ${ }^{38, c}$, M. Deák ${ }^{12}$, Y. de Boer ${ }^{12}$, B. Delcourt ${ }^{28}$, M. Del Degan ${ }^{41}$, J. Delvax ${ }^{4,5}$, E.A. De Wolf ${ }^{4,5}$, C. Diaconu $^{22}$, V. Dodonov ${ }^{14}$, A. Dossanov $^{27}$,

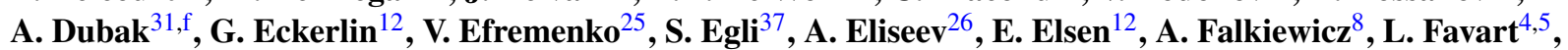
A. Fedotov ${ }^{25}$, R. Felst ${ }^{12}$, J. Feltesse ${ }^{11, h}$, J. Ferencei ${ }^{17}$, D.-J. Fischer ${ }^{12}$, M. Fleischer ${ }^{12}$, A. Fomenko ${ }^{26}$, E. Gabathuler ${ }^{19}$, J. Gayler ${ }^{12}$, S. Ghazaryan ${ }^{39}$, A. Glazov ${ }^{12}$, I. Glushkov ${ }^{40}$, L. Goerlich ${ }^{8}$, N. Gogitidze ${ }^{26}$, M. Gouzevitch ${ }^{12}$, C. Grab $^{41}$, T. Greenshaw ${ }^{19}$, B.R. Grell ${ }^{12}$, G. Grindhammer ${ }^{27}$, S. Habib ${ }^{13}$, D. Haidt ${ }^{12}$, C. Helebrant ${ }^{12}$, R.C.W. Henderson ${ }^{18}$,

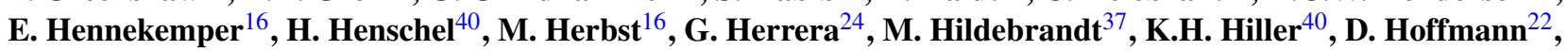

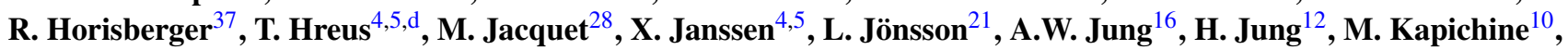
J. Katzy ${ }^{12}$, I.R. Kenyon ${ }^{3}$, C. Kiesling ${ }^{27}$, M. Klein ${ }^{19}$, C. Kleinwort ${ }^{12}$, T. Kluge $^{19}$, A. Knutsson ${ }^{12}$, R. Kogler $^{27}$, P. Kostka ${ }^{40}$, M. Kraemer ${ }^{12}$, K. Krastev ${ }^{12}$, J. Kretzschmar ${ }^{19}$, A. Kropivnitskaya ${ }^{25}$, K. Krüger $^{16}$, K. Kutak ${ }^{12}$, M.P.J. Landon ${ }^{20}$, W. Lange ${ }^{40}$, G. Laštovička-Medin ${ }^{31}$, P. Laycock ${ }^{19}$, A. Lebedev ${ }^{26}$, G. Leibenguth ${ }^{41}$,

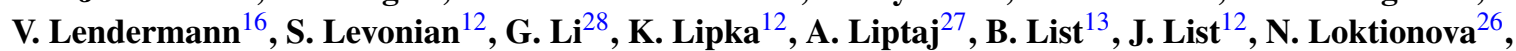
R. Lopez-Fernandez ${ }^{24}$, V. Lubimov ${ }^{25}$, A. Makankine ${ }^{10}$, E. Malinovski ${ }^{26}$, P. Marage ${ }^{4,5}$, Ll. Marti ${ }^{12}$, H.-U. Martyn ${ }^{1}$,

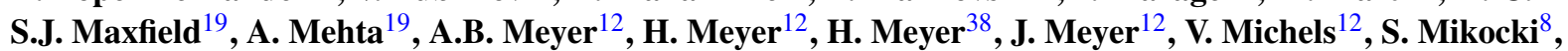
I. Milcewicz-Mika ${ }^{8}$, F. Moreau $^{29}$, A. Morozov ${ }^{10}$, J.V. Morris ${ }^{7}$, M.U. Mozer ${ }^{4,5}$, M. Mudrinic $^{2}$, K. Müller ${ }^{42}$,

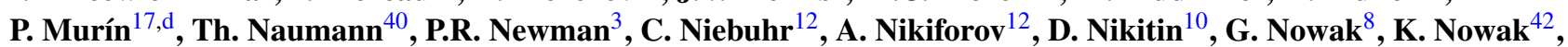
M. Nozicka ${ }^{12}$, B. Olivier ${ }^{27}$, J.E. Olsson ${ }^{12}$, S. Osman ${ }^{21}$, D. Ozerov ${ }^{25}$, V. Palichik ${ }^{10}$, I. Panagoulias ${ }^{12, b, v}$,

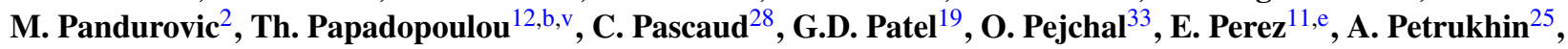
I. Picuric ${ }^{31}$, S. Piec ${ }^{40}$, D. Pitzl ${ }^{12}$, R. Plačakytè ${ }^{12}$, B. Pokorny ${ }^{13}$, R. Polifka ${ }^{33}$, B. Povh ${ }^{14}$, V. Radescu ${ }^{12}$, A.J. Rahmat ${ }^{19}$,

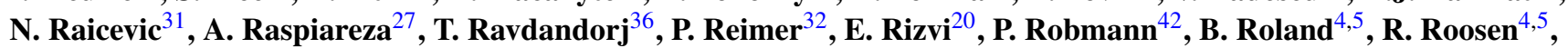
A. Rostovtsev ${ }^{25}$, M. Rotaru $^{6}$, J.E. Ruiz Tabasco ${ }^{23}$, Z. Rurikova ${ }^{12}$, S. Rusakov ${ }^{26}$, D. Š́lek ${ }^{33}$, D.P.C. Sankey ${ }^{7}$, M. Sauter ${ }^{41}$, E. Sauvan ${ }^{22}$, S. Schmitt ${ }^{12, a}$, L. Schoeffel ${ }^{11}$, A. Schöning ${ }^{15}$, H.-C. Schultz-Coulon ${ }^{16}$, F. Sefkow ${ }^{12}$, R.N. Shaw-West ${ }^{3}$, L.N. Shtarkov ${ }^{26}$, S. Shushkevich ${ }^{27}$, T. Sloan ${ }^{18}$, I. Smiljanic ${ }^{2}$, Y. Soloviev ${ }^{26}$, P. Sopicki ${ }^{8}$, D. South ${ }^{9}$, V. Spaskov ${ }^{10}$, A. Specka ${ }^{29}$, Z. Staykova ${ }^{12}$, M. Steder ${ }^{12}$, B. Stella ${ }^{34}$, G. Stoicea $^{6}$, U. Straumann ${ }^{42}$, D. Sunar ${ }^{4,5}$,

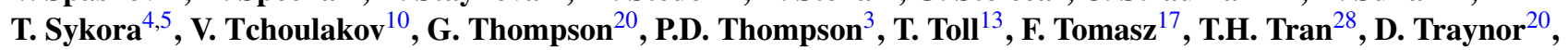

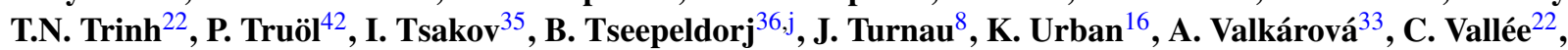
P. Van Mechelen ${ }^{4,5}$, A. Vargas Trevino ${ }^{12}$, Y. Vazdik ${ }^{26}$, S. Vinokurova $^{12}$, V. Volchinski ${ }^{39}$, M. von den Driesch ${ }^{12}$, D. Wegener ${ }^{9}$, Ch. Wissing ${ }^{12}$, E. Wünsch ${ }^{12}$, J. Žáček ${ }^{33}$, J. Zálešák ${ }^{32}$, Z. Zhang ${ }^{28}$, A. Zhokin ${ }^{25}$, T. Zimmermann ${ }^{41}$, H. Zohrabyan ${ }^{39}$, F. Zomer ${ }^{28}$, R. Zus ${ }^{6}$

\footnotetext{
${ }^{1}$ I. Physikalisches Institut der RWTH, Aachen, Germany

${ }^{2}$ Vinca Institute of Nuclear Sciences, Belgrade, Serbia

${ }^{3}$ School of Physics and Astronomy, University of Birmingham, Birmingham, UK ${ }^{1}$

${ }^{4}$ Inter-University Institute for High Energies ULB-VUB, Brussels, Belgium ${ }^{\mathrm{m}}$

${ }^{5}$ Universiteit Antwerpen, Antwerpen, Belgium ${ }^{\mathrm{m}}$

${ }^{6}$ National Institute for Physics and Nuclear Engineering (NIPNE), Bucharest, Romania

${ }^{7}$ Rutherford Appleton Laboratory, Chilton, Didcot, UK ${ }^{1}$

${ }^{8}$ Institute for Nuclear Physics, Cracow, Poland ${ }^{\text {n }}$

${ }^{9}$ Institut für Physik, TU Dortmund, Dortmund, Germany ${ }^{\mathrm{k}}$

${ }^{10}$ Joint Institute for Nuclear Research, Dubna, Russia

${ }^{11} \mathrm{CEA}, \mathrm{DSM} / \mathrm{Irfu}, \mathrm{CE}-$ Saclay, Gif-sur-Yvette, France

${ }^{12}$ DESY, Hamburg, Germany

${ }^{13}$ Institut für Experimentalphysik, Universität Hamburg, Hamburg, Germanyk

${ }^{14}$ Max-Planck-Institut für Kernphysik, Heidelberg, Germany
} 
${ }^{15}$ Physikalisches Institut, Universität Heidelberg, Heidelberg, Germany ${ }^{\mathrm{k}}$

${ }^{16}$ Kirchhoff-Institut für Physik, Universität Heidelberg, Heidelberg, Germany ${ }^{\mathrm{k}}$

${ }^{17}$ Institute of Experimental Physics, Slovak Academy of Sciences, Košice, Slovak Republic ${ }^{p}$

${ }^{18}$ Department of Physics, University of Lancaster, Lancaster, UK ${ }^{1}$

${ }^{19}$ Department of Physics, University of Liverpool, Liverpool, $\mathrm{UK}^{1}$

${ }^{20}$ Queen Mary and Westfield College, London, UK ${ }^{1}$

${ }^{21}$ Physics Department, University of Lund, Lund, Sweden ${ }^{\mathrm{q}}$

${ }^{22}$ CPPM, CNRS/IN2P3-Univ. Mediterranee, Marseille, France

${ }^{23}$ Departamento de Fisica Aplicada, CINVESTAV, Mérida, Yucatán, Mexico ${ }^{t}$

${ }^{24}$ Departamento de Fisica, CINVESTAV, México City, Mexico ${ }^{t}$

${ }^{25}$ Institute for Theoretical and Experimental Physics, Moscow, Russia ${ }^{\mathrm{u}}$

${ }^{26}$ Lebedev Physical Institute, Moscow, Russia ${ }^{\circ}$

${ }^{27}$ Max-Planck-Institut für Physik, München, Germany

${ }^{28}$ LAL, Univ. Paris-Sud, CNRS/IN2P3, Orsay, France

${ }^{29}$ LLR, Ecole Polytechnique, CNRS/IN2P3, Palaiseau, France

${ }^{30}$ LPNHE, Universités Paris VI and VII, CNRS/IN2P3, Paris, France

${ }^{31}$ Faculty of Science, University of Montenegro, Podgorica, Montenegro ${ }^{\circ}$

${ }^{32}$ Institute of Physics, Academy of Sciences of the Czech Republic, Praha, Czech Republic ${ }^{\mathrm{r}}$

${ }^{33}$ Faculty of Mathematics and Physics, Charles University, Praha, Czech Republic ${ }^{\mathrm{r}}$

${ }^{34}$ Dipartimento di Fisica Università di Roma Tre and INFN Roma 3, Roma, Italy

${ }^{35}$ Institute for Nuclear Research and Nuclear Energy, Sofia, Bulgaria ${ }^{\circ}$

${ }^{36}$ Institute of Physics and Technology of the Mongolian Academy of Sciences, Ulaanbaatar, Mongolia

${ }^{37}$ Paul Scherrer Institut, Villigen, Switzerland

${ }^{38}$ Fachbereich C, Universität Wuppertal, Wuppertal, Germany

${ }^{39}$ Yerevan Physics Institute, Yerevan, Armenia

${ }^{40}$ DESY, Zeuthen, Germany

${ }^{41}$ Institut für Teilchenphysik, ETH, Zürich, Switzerland ${ }^{\mathrm{s}}$

${ }^{42}$ Physik-Institut der Universität Zürich, Zürich, Switzerland ${ }^{\mathrm{s}}$

Received: 29 October 2009 / Revised: 18 December 2009 / Published online: 28 January 2010

(C) Springer-Verlag / Società Italiana di Fisica 2010

a e-mail: sschmitt@mail.desy.de, kruegerk@mail.desy.de

${ }^{\mathrm{b}}$ Also at Physics Department, National Technical University, Zografou Campus, 15773 Athens, Greece

${ }^{\mathrm{c}}$ Also at Rechenzentrum, Universität Wuppertal, Wuppertal, Germany

${ }^{\mathrm{d}}$ Also at University of P.J. Šafárik, Košice, Slovak Republic

eAlso at CERN, Geneva, Switzerland

${ }^{\mathrm{f}}$ Also at Max-Planck-Institut für Physik, München, Germany

${ }^{g}$ Also at Comenius University, Bratislava, Slovak Republic

${ }^{\mathrm{h}}$ Also at DESY and University Hamburg, Helmholtz Humboldt Research Award

${ }^{\mathrm{i}}$ Also at Faculty of Physics, University of Bucharest, Bucharest, Romania

${ }^{\mathrm{j}}$ Also at Ulaanbaatar University, Ulaanbaatar, Mongolia

${ }^{\mathrm{k}}$ Supported by the Bundesministerium für Bildung und Forschung, FRG, under contract numbers 05H09GUF, 05H09VHC, 05H09VHF, 05H16PEA

${ }^{1}$ Supported by the UK Science and Technology Facilities Council, and formerly by the UK Particle Physics and Astronomy Research Council

${ }^{\mathrm{m}}$ Supported by FNRS-FWO-Vlaanderen, IISN-IIKW and IWT and by Interuniversity Attraction Poles Programme, Belgian Science Policy

${ }^{\mathrm{n}}$ Partially Supported by Polish Ministry of Science and Higher Education, grant PBS/DESY/70/2006

${ }^{\circ}$ Supported by the Deutsche Forschungsgemeinschaft

PSupported by VEGA SR grant No. 2/7062/27
Abstract The production of prompt photons is measured in the photoproduction regime of electron-proton scattering at HERA. The analysis is based on a data sample corresponding to a total integrated luminosity of $340 \mathrm{pb}^{-1}$ collected by the $\mathrm{H} 1$ experiment. Cross sections are measured for photons with transverse momentum and pseudorapidity in the range $6<E_{T}^{\gamma}<15 \mathrm{GeV}$ and $-1.0<\eta^{\gamma}<2.4$, respectively. Cross sections for events with an additional jet are measured as a function of the transverse energy and pseudorapidity of the jet, and as a function of the fractional momenta $x_{\gamma}$ and $x_{p}$ carried by the partons entering the hard scattering process. The correlation between the photon and the jet is also studied. The results are compared with QCD predictions based on the collinear and on the $k_{T}$ factorization approaches.

qSupported by the Swedish Natural Science Research Council

${ }^{\mathrm{r}}$ Supported by the Ministry of Education of the Czech Republic under the projects LC527, INGO-1P05LA259 and MSM0021620859

${ }^{\mathrm{s}}$ Supported by the Swiss National Science Foundation

${ }^{\text {t}}$ Supported by CONACYT, Mexico, grant 48778-F

unussian Foundation for Basic Research (RFBR), grant No. 1329.2008.2

${ }^{\mathrm{v}}$ This project is co-funded by the European Social Fund (75\%) and National Resources (25\%)—(EPEAEK II)—PYTHAGORAS II 

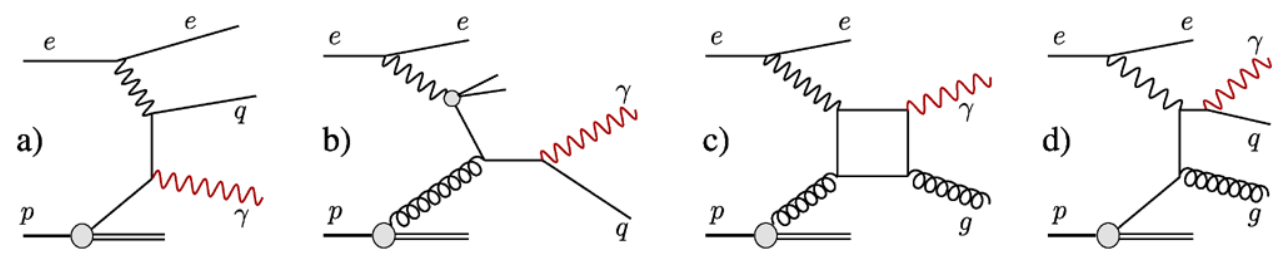

Fig. 1 Examples of diagrams for the production of prompt photons in photoproduction: (a) direct and (b) resolved interaction with a parton from the proton, (c) box diagram and (d) radiation of a photon from an outgoing quark

\section{Introduction}

Isolated photons emerging from the hard subprocess $e p \rightarrow$ $e \gamma X$, so called prompt photons, are a powerful probe of the underlying dynamics, complementary to jets. Production of isolated photons with high transverse momentum can be calculated in perturbation theory. High energy electronproton scattering is dominated by so-called photoproduction processes, in which a beam lepton emits a quasi-real photon which either interacts directly with the proton (direct process) or fluctuates into partons which then participate in the hard scattering process (resolved process). In prompt photon production, the direct process is sensitive to the quark content of the proton through the Compton scattering of the exchanged photon with a quark $(\gamma q \rightarrow \gamma q)$ as depicted in Fig. 1(a). The resolved process $(q g \rightarrow \gamma q)$ is sensitive to the partonic structure of both the photon and the proton. A typical diagram is shown in Fig. 1(b). Figures 1(c) and 1(d) show typical higher order diagrams.

The H1 collaboration has previously measured prompt photon cross sections in photoproduction [1] and in deep inelastic scattering (DIS) [2]. The ZEUS collaboration has also reported measurements of prompt photon production [3-5]. Both experiments found that in photoproduction the inclusive prompt photon cross section is underestimated by next-to-leading order (NLO) QCD calculations [6-8], while there is reasonable agreement for events with a prompt photon and a jet (photon plus jet). In DIS, a leading order QCD calculation [9] significantly underestimates the production of isolated photons and of photons plus jets. NLO predictions [10] are only available for the latter and also underestimate the cross section.

This paper presents results of a measurement of prompt photons in photoproduction. The data used for the measurement were collected with the $\mathrm{H} 1$ detector in the period from 2004 to 2007 and correspond to a total integrated luminosity of $340 \mathrm{pb}^{-1}$. This amounts to an increase in statistics by a factor of three compared to the previous measurement [1]. During this data taking period HERA collided positrons or electrons $^{1}$ of energy $E_{e}=27.6 \mathrm{GeV}$ with protons of energy

\footnotetext{
${ }^{1}$ Unless otherwise stated, the term electron refers to both the electron and the positron.
}

$E_{p}=920 \mathrm{GeV}$ corresponding to a center-of-mass energy of $\sqrt{s}=319 \mathrm{GeV}$.

Isolated photons with transverse energy $6<E_{T}^{\gamma}<$ $15 \mathrm{GeV}$ and pseudorapidity ${ }^{2}-1.0<\eta^{\gamma}<2.4$ are measured in events with the inelasticity $y$ in the range $0.1<y<0.7$. This extends the phase space of previous measurements at HERA towards larger pseudorapidities of the photon and to smaller event inelasticities.

The main background is due to photons produced in hadron decays. For its discrimination from prompt photons, various shower shape variables are used. Differential cross sections are presented as a function of the transverse energy and pseudorapidity of the photon. For the photon plus jet sample, differential cross sections are measured as a function of transverse energy and pseudorapidity of the photon and the jet and the momentum fractions $x_{\gamma}$ and $x_{p}$ carried by the participating parton in the photon and the proton, respectively. Azimuthal angle and transverse momentum correlations between the photon and the jet are also studied. The cross sections are compared to QCD calculations based on collinear factorization in NLO $[6,7]$ and to calculations based on the $k_{T}$ factorization approach [11].

\section{Theoretical predictions}

The calculation by Fontannaz, Guillet and Heinrich (FGH) $[6,7]$ based on the collinear factorization approach includes the leading order direct and resolved processes $\gamma q \rightarrow \gamma q$ and their NLO corrections. Besides the production of a prompt photon in the hard interaction, photons may originate from the fragmentation of a high momentum quark or gluon in the final state. The fragmentation process, described by a fragmentation function, is included in the calculation as well as the direct box diagram as shown in Fig. 1(c). The contribution from quark to photon fragmentation to the total cross section of isolated photons is at the level of $4 \%$. The contribution from the box diagram amounts to about $10 \%$ on average. The calculation uses the parton

\footnotetext{
${ }^{2}$ The pseudorapidity is related to the polar angle $\theta$ as $\eta=$ $-\ln \tan (\theta / 2)$, where $\theta$ is measured with respect to the direction of the outgoing proton beam (forward direction).
} 
density functions (PDFs) CTEQ6L [12] for the proton and AFG04[13] for the photon. The scales for renormalization $\mu_{R}$ and factorization $\mu_{F}$, are chosen to be $\mu_{R}=\mu_{F}=E_{T}^{\gamma}$. The NLO corrections to the LO cross section are significant for the inclusive sample. They increase the predicted cross section by a factor 1.15-1.42, the corrections being largest at low $E_{T}^{\gamma}$ and large $\eta^{\gamma}$. For the photon plus jet sample the corrections are much smaller and below $10 \%$ on average.

The leading order predictions of Lipatov and Zotov (LZ) [11] are based on the $k_{T}$ factorization approach. The calculation uses the unintegrated quark and gluon densities of the photon and the proton using the KimberMartin-Ryskin (KMR) prescription [14] with the GRV parameterizations for the collinear quark and gluon densities [15, 16]. The $k_{T}$ factorization approach is expected to account for the main part of the collinear higher order QCD corrections [11]. Direct and resolved processes are considered in the calculation, but contributions from fragmentation and from the box diagram are neglected.

To ensure isolation of the photon, the total transverse energy within a cone of radius one in the pseudorapidity - azimuthal angle plane surrounding the prompt photon, excluding its own energy, is required to be below $10 \%$ of $E_{T}^{\gamma}$ in both calculations. This requirement slightly differs from the one used in the data analysis as described in Sect. 4.1.

The theoretical predictions are compared to the data after a correction for multi parton interactions, for hadronization effects and for the different definition of the isolation of the photon. The total correction factors $f_{\text {corr }}$ are determined with the signal MC described below as the ratios of the cross sections on hadron level with multi-parton interactions and the data isolation criteria, to the cross sections on parton level without multi-parton interactions and using the cone cut for the isolation of the photon. The correction factors are calculated for each bin using the event generators PYTHIA [17] and HERWIG[18] which have a different model for hadronization. The arithmetic means of the two correction factors are used, while half of the difference between the two models is taken as the error. The correction factors for the total inclusive cross section range from 0.84 to 0.99 with an average of 0.9 . They are largest for low $E_{T}^{\gamma}$ and in the forward direction, where the photon isolation is most sensitive to hadronization and to multi-parton interactions. The uncertainty of the corrections is typically $8 \%$.

The leading order MC generator PYTHIA 6.2 [17] is used in this analysis for the prediction of the signal. The simulation of multi-parton interactions $[19,20]$ is included. The hard partonic interaction is calculated in LO QCD and higher order QCD radiation is modeled using initial and final state parton showers in the leading log approximation [21]. The fragmentation into hadrons is simulated in PYTHIA by the Lund string model [22]. The simulated signal contains contributions from direct (Fig. 1(a)) and resolved (Fig. 1(b)) production of prompt photons including QED radiation. In addition, processes with two hard partons in the final state (Fig. 1(d)) are simulated. The simulations use the parton densities CTEQ6L [12] for the proton and SASG-1D [23] for the photon. Different parton density functions for the proton (CTEQ5L [24] and MRST04 [25]) and the photon (GRV [15] and AFG04) are used to estimate the influence of the parton densities on the predicted cross section, which varies by at most $10 \%$, mainly due to changes of the proton PDF. The multi-parton interactions reduce the total inclusive cross section by $6 \%$ on average. The uncertainty of the correction for multi-parton interactions is estimated by changing the default parameter for the effective minimum transverse momentum for multi-parton interactions in PYTHIA (PARP(81)) from 1.9 to $1.6 \mathrm{GeV}$ and $2.2 \mathrm{GeV}$, respectively.

To estimate the uncertainty of the hadronization correction, the HERWIG [18] generator is also used to model the prompt photon signal. HERWIG simulates the fragmentation into hadrons through the decay of colorless parton clusters.

Background to the analysis of prompt photons mainly arises from energetic photons from the decay of hadrons like $\pi^{0}$ and $\eta$ in photoproduction events, which constitute more than $90 \%$ of the total background prediction. Direct and resolved photoproduction of di-jet events used to study the background is simulated with PYTHIA.

All generated events are passed through a GEANT [26] based simulation of the $\mathrm{H} 1$ detector which takes into account the different data taking periods, and are subject to the same reconstruction and analysis chain as the data.

\section{H1 detector}

A detailed description of the $\mathrm{H} 1$ detector can be found in $[27,28]$. In the following, only detector components relevant to this analysis are briefly discussed. The origin of the H1 coordinate system is the nominal $e p$ interaction point, with the direction of the proton beam defining the positive $z$-axis (forward direction). Transverse momenta are measured in the $x-y$ plane. Polar $(\theta)$ and azimuthal $(\phi)$ angles are measured with respect to this reference system.

In the central region $\left(15^{\circ}<\theta<165^{\circ}\right)$ the interaction point is surrounded by the central tracking system (CTD), which consists of a silicon vertex detector [29] and drift chambers all operated within a solenoidal magnetic field of $1.16 \mathrm{~T}$. The forward tracking detector and the backward proportional chamber measure tracks of charged particles at smaller $\left(7^{\circ}<\theta<25^{\circ}\right)$ and larger $\left(155^{\circ}<\theta<175^{\circ}\right)$ polar angles than the central tracker, respectively. In each event the $e p$ interaction vertex is reconstructed from the charged tracks. In the polar angular region $\left(11^{\circ}<\theta<169^{\circ}\right)$ an additional track signature is obtained from a set of five cylindrical multi-wire proportional chambers (CIP2k) [30]. 
The liquid argon (LAr) sampling calorimeter [31] surrounds the tracking chambers. It has a polar angle coverage of $4^{\circ}<\theta<154^{\circ}$ and full azimuthal acceptance. It consists of an inner electromagnetic section with lead absorbers and an outer hadronic section with steel absorbers. The calorimeter is divided into eight wheels along the beam axis. The electromagnetic and the hadronic sections are highly segmented in the transverse and the longitudinal directions. Electromagnetic shower energies are measured with a precision of $\sigma(E) / E=12 \% / \sqrt{E / \mathrm{GeV}} \oplus 1 \%$ and hadronic energies with $\sigma(E) / E=50 \% / \sqrt{E / \mathrm{GeV}} \oplus 2 \%$, as determined in test beam experiments $[32,33]$. In the backward region $\left(153^{\circ}<\theta<178^{\circ}\right)$, particle energies are measured by a leadscintillating fibre spaghetti calorimeter (SpaCal) [34].

The luminosity is determined from the rate of the BetheHeitler process $e p \rightarrow e p \gamma$, measured using a photon detector located close to the beam pipe at $z=-103 \mathrm{~m}$.

The LAr calorimeter provides the trigger [35] for the events in this analysis. The hardware trigger is complemented by a software trigger requiring an electromagnetic cluster in the LAr calorimeter with a transverse energy $E_{T}^{\gamma}>5 \mathrm{GeV}$. The combined trigger efficiency is about $85 \%$ at $E_{T}^{\gamma}$ of $6 \mathrm{GeV}$ rising to above $95 \%$ for $E_{T}^{\gamma}>7 \mathrm{GeV}$.

\section{Experimental method}

\subsection{Event selection and reconstruction}

Events are selected with a photon candidate in the LAr calorimeter of transverse energy $6<E_{T}^{\gamma}<15 \mathrm{GeV}$ and pseudorapidity $-1.0<\eta^{\gamma}<2.4$. Photon candidates are defined as compact clusters in the electromagnetic section of the LAr calorimeter with no matching signals in the CIP2k. The CIP2k veto rejects candidates, if there is a signal in at least two layers of the CIP2k close to the expected hit position. In addition, a track veto is applied for $\theta>45^{\circ}$. It rejects candidates, if a track in the CTD extrapolated to the LAr calorimeter front face matches the electromagnetic cluster with a distance of closest approach to the cluster's barycenter of less than $15 \mathrm{~cm}$. Photon candidates are also rejected if they are close to inactive regions between calorimeter modules.

Neutral current (NC) deep-inelastic scattering (DIS) events are suppressed by rejecting events with an electron candidate not previously identified as photon candidate. Electron candidates are defined as compact electromagnetic clusters in the SpaCal or in the LAr calorimeter. In the LAr calorimeter the candidates are required to have an associated track with a distance of closest approach of less than $12 \mathrm{~cm}$. The electron suppression restricts the sample to NC events where the scattered electron escapes along the beam pipe in the negative $z$ direction. The low electron scattering angle of such events corresponds to virtualities of the exchanged photon in the range $Q^{2}<4 \mathrm{GeV}^{2}$. In photoproduction the inelasticity $y$ is expressed as $y=W^{2} / s$, where $W$ is the $\gamma p$ center of mass energy. In this analysis $y$ is evaluated as $y_{h}=\sum\left(E-p_{z}\right) / 2 E_{e}$, where the sum runs over all measured final state particles with energy $E$ and longitudinal momentum $p_{z}$. The inelasticity is restricted to $0.1<y_{h}<0.7$. The cut at low $y_{h}$ removes residual beam gas background and the higher cut on $y_{h}$ removes background from DIS events including events with prompt photons and events where the scattered electron is misidentified as a photon. This background is below $1.5 \%$ in the final sample and is considered as a systematic uncertainty.

In order to remove background events from non-ep sources, at least two tracks are required in the central tracker, assuring a good reconstruction of the longitudinal event vertex position which is required to be within $40 \mathrm{~cm}$ around the nominal interaction point. In addition, topological filters and timing vetoes are applied to remove cosmic muons and beam induced background.

The shape of the photon cluster candidate is used to further reduce the background. The transverse ${ }^{3}$ radius $R_{T}$ of the photon candidate is defined as the square root of the second central transverse moment $R_{T}=\sqrt{\mu_{2}}$, where the $k$ 'th central transverse moment of the calorimeter cells distribution is given by $\mu_{k}=\left\langle|\vec{r}-\langle\vec{r}\rangle|^{k}\right\rangle$. Here, $\vec{r}$ is the transverse projection of a cell position and the averages are calculated taking into account the cell energies as weight factors. The requirement $R_{T}<6 \mathrm{~cm}$ reduces background from neutral hadrons that decay into multiple photons. In most cases such decay photons are merged into one electromagnetic cluster, which tends to have a wider transverse spread than that of a single photon.

For events where a second electromagnetic cluster is found, the invariant mass $M_{\gamma \gamma}$ of the photon candidate cluster, combined with the closest neighboring electromagnetic cluster with an energy above $80 \mathrm{MeV}$, is reconstructed. Photon candidates from $\pi^{0}$ decays where the two decay photons are reconstructed in separate clusters are rejected requiring $M_{\gamma \gamma}>300 \mathrm{MeV}$.

Tracks and calorimeter energy deposits not previously identified as photon candidate are used to form combined cluster-track objects. The photon candidate and the clustertrack objects are combined into massless jets using the inclusive $k_{T}$ algorithm [36] with the separation parameter $R_{0}$ set to 1 . Jets are reconstructed in the pseudorapidity range $-2.0<\eta^{\text {jet }}<3.0$ with a transverse momentum of $E_{T}^{\mathrm{jet}}>4 \mathrm{GeV}$. Due to the harder kinematical cuts for the photon candidate there is always a jet containing the photon candidate called the photon-jet. All other jets are classified

\footnotetext{
${ }^{3}$ In the context of the cluster shape analysis the transverse plane is defined as perpendicular to the direction of the photon candidate.
} 
Fig. 2 Distributions of (a) the inelasticity $y_{h},(\mathbf{b})$ the transverse energy $E_{T}^{\gamma}$ of the photon, (c) the isolation parameter $z$ and (d) $\eta^{\text {jet }}$ for events with prompt photon candidates. Data are shown as points with error bars indicating the statistical error.

The signal prediction from PYTHIA for prompt photons is shown as dark full line, and the contribution of direct interactions as a dotted line. The background as simulated with PYTHIA is shown as a dashed line. The signal and background contributions are scaled on average by a factor 1.5 and 1.7 , respectively. The sum of the scaled signal and background is shown as the light gray histogram. The vertical dashed lines indicate the kinematic region of the cross section measurement
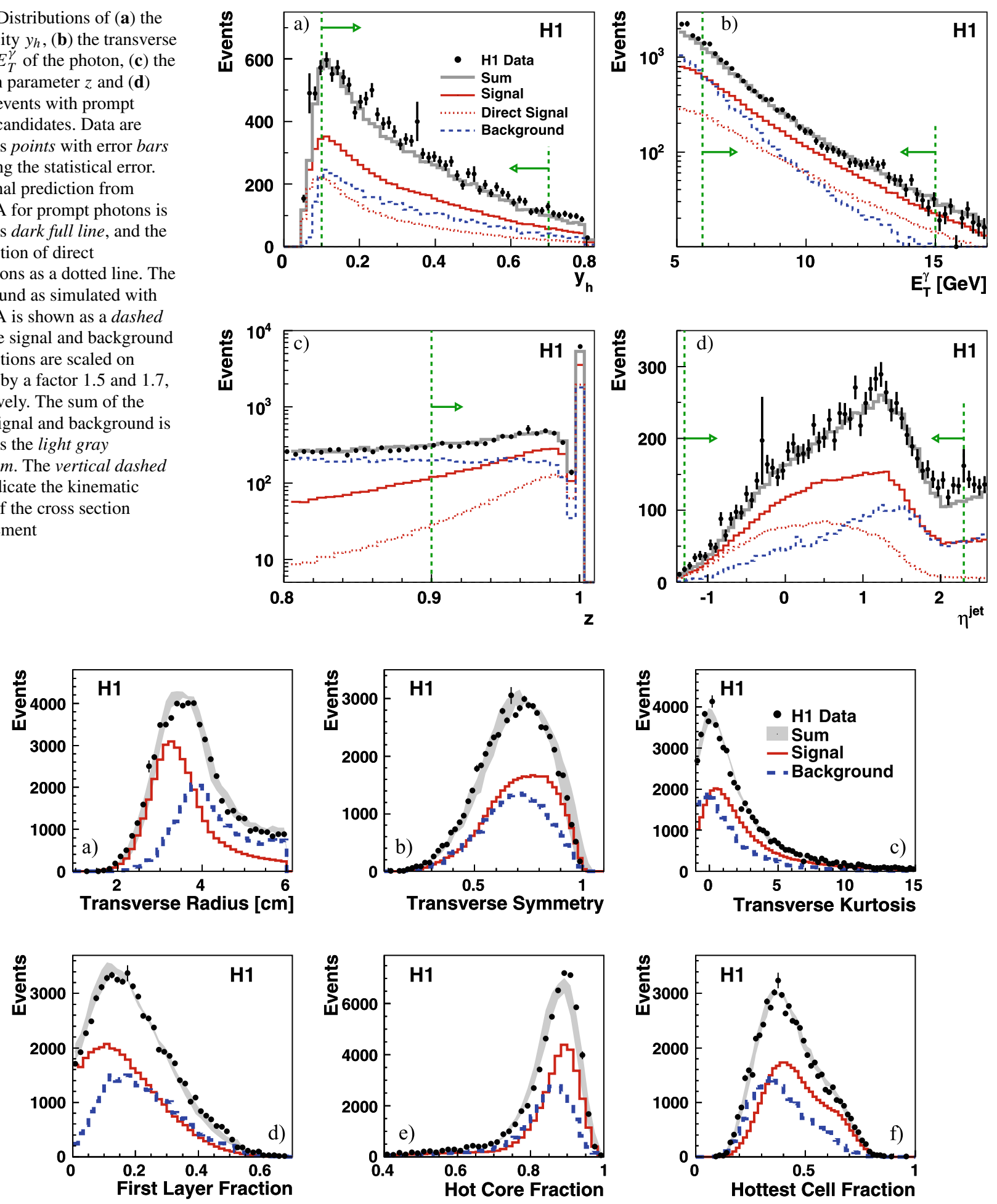

Fig. 3 Distributions of the shower shape variables that are used to define the discriminant for isolated photon identification: (a) transverse radius, (b) transverse symmetry, (c) transverse kurtosis, (d) the fraction of energy in the first layer, (e) the fraction of energy in the hot core of the cluster and (f) the fraction of energy in the hottest cell of the cluster for all the photon candidates. Data are shown as points with error bars for the statistical error. The expectation from PYTHIA for the signal (background) is shown as a full (dashed) line. The signal and background contributions are scaled on average by a factor 1.5 and 1.7, respectively. The shaded band shows the sum of the MC predictions. The width of the band corresponds to the systematic uncertainty assigned to the description of the shower shapes 
Fig. 4 The distribution of the discriminant $D$ for $0.94<\eta^{\gamma}<1.42$ in four different $E_{T}^{\gamma}$ bins. $D$ is used in the identification of prompt photon candidates for events that have passed the event selection. Data are shown as points with error bars for the statistical error. The expectation from PYTHIA for the signal (background) is shown as a full (dashed) line. The signal and background contributions are scaled by a factor 1.5 and 1.7 on average, respectively. The shaded band shows the sum of the MC predictions with the systematic uncertainty from the description of the shower shapes
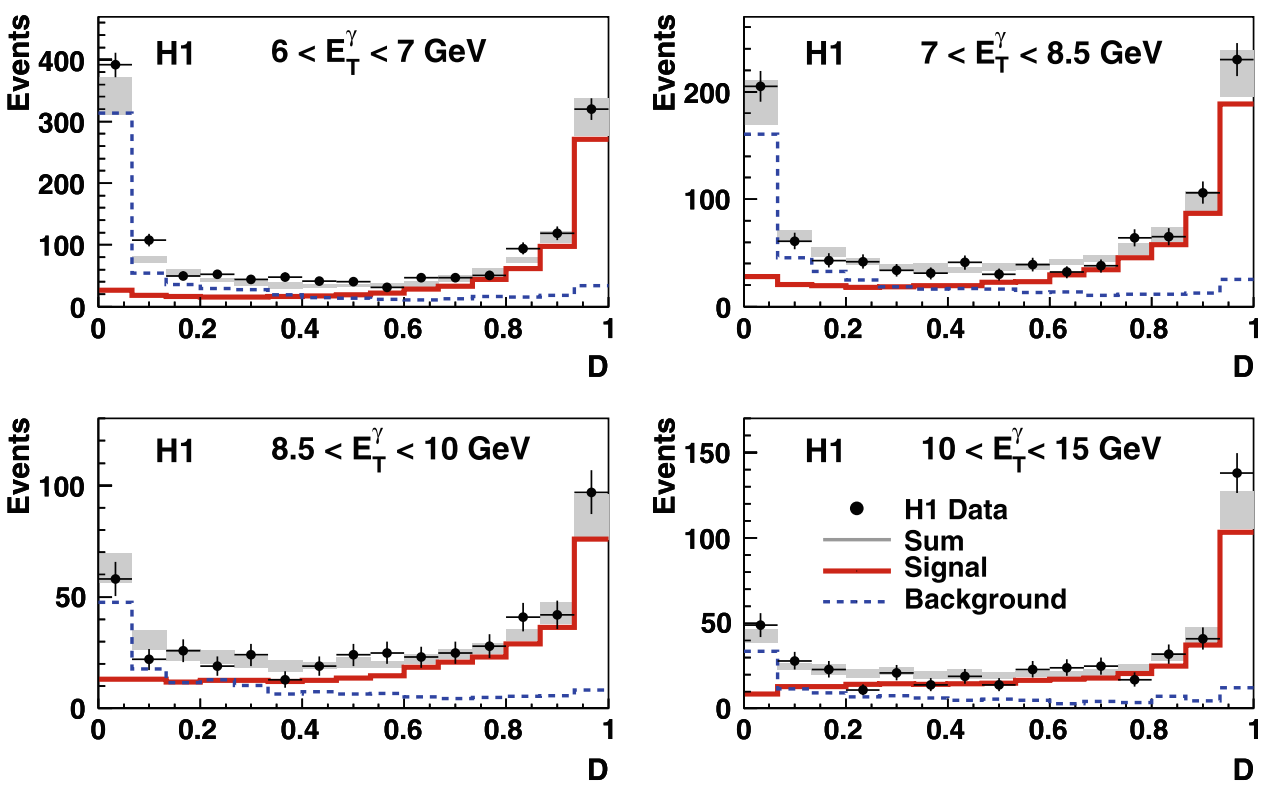

as hadronic jets. To ensure isolation of the photon, the fraction $z=E_{T}^{\gamma} / E_{T}^{\gamma-\text { jet }}$ of the transverse energy of the photonjet carried by the photon candidate has to be larger than 0.9 . Here, $E_{T}^{\gamma-\text { jet }}$ is the transverse energy of the photon-jet. This isolation requirement largely suppresses background from photons produced in the hadron decay cascade. Only events with exactly one isolated photon candidate are accepted.

For the photon plus jet sample, events are selected with a photon candidate and at least one hadronic jet with $-1.3<$ $\eta^{\text {jet }}<2.3$. If more than one hadronic jet is selected, the one with the highest $E_{T}^{\text {jet }}$ is used.

Four additional observables are defined for the photon plus jet sample which are sensitive to the underlying partonic process:

- The estimators $x_{\gamma}^{\mathrm{LO}}$ and $x_{p}^{\mathrm{LO}}$, which in the LO approximation correspond to the longitudinal momentum fractions of the partons in the photon and the proton, respectively, are defined as

$$
\begin{aligned}
& x_{\gamma}^{\mathrm{LO}}=\frac{E_{T}^{\gamma}\left(e^{-\eta^{\mathrm{jet}}}+e^{-\eta^{\gamma}}\right)}{\left(2 y_{h} E_{e}\right)} \text { and } \\
& x_{p}^{\mathrm{LO}}=\frac{E_{T}^{\gamma}\left(e^{\eta^{\mathrm{jet}}}+e^{\eta^{\gamma}}\right)}{\left(2 E_{p}\right)} .
\end{aligned}
$$

These definitions [37, 38] reduce infrared sensitivity for $x_{\gamma} \rightarrow 1$ compared to the conventional definition of $x_{\gamma}=\left(E_{T}^{\gamma} e^{-\eta^{\gamma}}+E_{T}^{\mathrm{jet}} e^{-\eta^{\mathrm{jet}}}\right) /\left(2 y E_{e}\right)$. The above definitions make use of the energy of the photon only, which has a better resolution than the energy of the jet. However, $x_{\gamma}^{\mathrm{LO}}$ and $x_{p}^{\mathrm{LO}}$ may become larger than unity.

- Two observables $p_{\perp}$ and $\Delta \Phi$ describe the transverse correlation between the photon and the jet, $\Delta \Phi$ is the azimuthal difference between the photon and the jet, and $p_{\perp}$ is the photon momentum component perpendicular to the jet direction in the transverse plane

$$
p_{\perp} \equiv \frac{\left|\vec{p}_{T}^{\gamma} \times \vec{p}_{T}^{\text {jet }}\right|}{\left|\vec{p}_{T}^{\text {jet }}\right|}=E_{T}^{\gamma} \cdot \sin \Delta \Phi .
$$

At leading order the prompt photon and the jet are backto-back and $p_{\perp}$ equals zero for direct processes. The observable $\Delta \Phi$ is strongly correlated with $p_{\perp}$, but is less sensitive to the energies of the photon and the jet.

The $y_{h}, E_{T}^{\gamma}, z$ and $\eta^{\text {jet }}$ distributions of events with an isolated photon candidate are shown in Fig. 2 together with the MC predictions from PYTHIA for the signal and the background. The signal (background) prediction is scaled by a factor 1.5 (1.7) on average. The scaling factors vary as a function of $\eta$ as suggested by the cross section measurement (Sect. 5). In all distributions the data are described within errors by the scaled MC predictions. At this stage of the analysis there is still a significant contribution of background from the decay products of neutral mesons.

\subsection{Photon signal extraction}

The photon signal is extracted from the sample with photon candidates by means of a shower shape analysis based on the method described in [2, 39]. It uses the following six shower shape variables calculated from the measurements of the individual cells composing the cluster:

- The transverse radius of the cluster, $R_{T}$.

- The transverse symmetry, which is the ratio of the spread of the transverse cell distributions along the two principal axes. Single photon clusters are expected to be more symmetric than multi-photon clusters. 
- The transverse kurtosis, defined as $K_{T}=\mu_{4} /\left(\mu_{2}\right)^{2}-3$, with $\mu_{2}$ and $\mu_{4}$ the second and the fourth moment of the transverse energy distribution.

- The first layer fraction, defined as the fraction of the cluster's energy detected in the first calorimeter layer.

- The hot core fraction, being the fraction of the energy of the electromagnetic cluster contained in the hot core of the cluster. It is defined as the energy fraction in four to twelve contiguous cells in the first two calorimeter layers, depending on the polar angle. The cells include the most energetic cell and are chosen to maximize the energy.

- The hottest cell fraction, which is the fraction of the energy of the electromagnetic cluster contained in the cell with the largest energy deposit.

The distributions of the shower shape variables are shown in Fig. 3 for the prompt photon candidates with the kinematic cuts as defined above. The shaded band shows the systematic uncertainty assigned to the description of the shower shapes as described in Sect. 4.4. The data are compared with the sum of the background and the signal MC distributions, which describe the data within the systematic error.

In order to discriminate between signal and background, probability density functions $p_{\gamma}^{i}$ for the signal and $p_{b g}^{i}$ for the background are defined for each of the six shower shape variables $i$. Simulated events for the signal and the background are used to determine $p_{\gamma}^{i}$ and $p_{b g}^{i}$. The photon and background probability densities are taken as the product of the respective shower shape densities with the method described in [40]. For each event a discriminator $D$ is formed. It is defined as the photon probability density divided by the sum of the probability densities for photons and background. Figure 4 shows an example of the discriminator distribution for the range $0.94<\eta^{\gamma}<1.42$ and four different bins in $E_{T}^{\gamma}$. The discriminator has in general larger values for prompt photons than for the decay photons. The separation power is decreasing with increasing $E_{T}^{\gamma}$. The sum of the MC predictions describes the data within the systematic uncertainty of the shower shapes.

Additional event samples are used for the determination of systematic errors related to the cluster shapes. The first sample, containing Bethe Heitler events, $e p \rightarrow e \gamma p$, consists of events with an electron reconstructed in the LAr calorimeter, a photon in the SpaCal and nothing else in the detector. The second, complementary sample, in addition containing deeply-virtual Compton scattering [46] events, is selected by requiring an electron in the $\mathrm{SpaCal}$, a photon in the LAr calorimeter and no other particle in the detector. These independent event selections, denoted BH and DVCS respectively, provide a clean sample of electromagnetic clusters at low transverse energies in the LAr calorimeter and are used to study the description of the shower shapes of the photons. A third sample is used to monitor the description of the shower shapes of clusters initiated by the decay
Table 1 Phase space for the measurement of prompt photon cross sections. Kinematics are defined in the H1 laboratory frame

H1 prompt photon phase space

\begin{tabular}{ll}
\hline Inclusive & $6<E_{T}^{\gamma}<15 \mathrm{GeV}$ \\
cross section & $-1.0<\eta^{\gamma}<2.4$ \\
& $z=E_{T}^{\gamma} / E_{T}^{\gamma-\text { jet }}>0.9$ \\
& $Q^{2}<1 \mathrm{GeV}^{2}$ \\
& $0.1<y<0.7$ \\
\hline Jet definition & $E_{T}^{\text {jet }}>4.5 \mathrm{GeV}$ \\
& $-1.3<\eta^{\text {jet }}<2.3$ \\
\hline
\end{tabular}

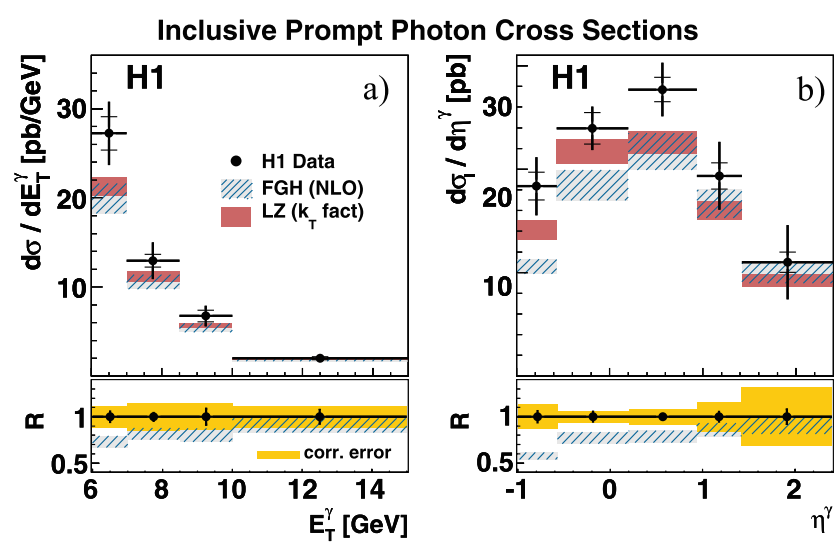

Fig. 5 Inclusive differential prompt photon cross sections (a) $\mathrm{d} \sigma / \mathrm{d} E_{T}^{\gamma}$ and (b) $\mathrm{d} \sigma / \mathrm{d} \eta^{\gamma}$ in the kinematic range specified in Table 1 . The inner error bars on the data points indicate the uncorrelated error including the statistical error, the full error bars contain in addition the correlated errors added in quadrature. The data are compared to a QCD calculation based on the collinear factorization in $\mathrm{NLO}(\mathrm{FGH})[6,7]$ and to a QCD calculation based on the $k_{T}$ factorization approach (LZ) [11]. For each plot the lower figure shows the ratio of NLO QCD to the measured cross section $\left(R=\sigma_{\mathrm{FGH}} / \sigma\right)$ as a hatched band. The width of this band shows the uncertainty from the NLO calculation only. The data points are shown at $R=1$ and their bars indicate the experimental uncorrelated uncertainty. The correlated experimental uncertainty of the data is indicated by the shaded area

of neutral hadrons. This sample, denoted BG, is background enhanced by selecting events with the inverted isolation criteria $z=E_{T}^{\gamma} / E_{T}^{\gamma-\text { jet }}<0.9$ and no cut on the transverse radius of the photon candidate.

\subsection{Cross section determination}

A regularized unfolding procedure [41-45] is used to relate distributions $\vec{y}_{\text {rec }}$ of reconstructed variables (input bins) to distributions $\vec{x}_{\text {true }}$ of variables on hadron level (output bins), to determine the fractions of signal and background and to correct the data for the detector acceptance. The unfolding matrix A relates the two vectors, $\mathbf{A} \vec{x}_{\text {true }}=\vec{y}_{\text {rec }}$. Further details on the method can be found in [45] and are summarized in Appendix. 
Table 2 Bin averaged differential cross sections for inclusive prompt photon production as a function of $\eta^{\gamma}$ and $E_{T}^{\gamma}$ in the kinematic range specified in Table 1 . The bin ranges, the differential cross section values, the uncorrelated and correlated uncertainties, and $f_{\text {corr }}$ are listed, where $f_{\text {corr }}$ denotes the correction factor applied to the theoretical cross sections. It corrects for multi-parton interactions, hadronization and the different algorithm used for the isolation cut

\begin{tabular}{lclll}
\hline H1 inclusive prompt photon cross sections & & & \\
\hline $\begin{array}{l}E_{T}^{\gamma} \\
{[\mathrm{GeV}]}\end{array}$ & $\mathrm{d} \sigma / \mathrm{d} E_{T}^{\gamma}$ & $\begin{array}{c}\text { Uncorr. } \\
{[\mathrm{pb} / \mathrm{GeV}]}\end{array}$ & Corr. & $f_{\text {corr }}$ \\
\hline$[6.0,7.0]$ & 27.24 & \pm 1.86 & \pm 3.04 & $0.88_{-0.04}^{+0.02}$ \\
{$[7.0,8.5]$} & 12.94 & \pm 0.71 & \pm 1.94 & $0.89_{-0.04}^{+0.02}$ \\
{$[8.5,10.0]$} & 6.74 & \pm 0.65 & \pm 0.95 & $0.93_{-0.04}^{+0.02}$ \\
{$[10.0,15.0]$} & 2.02 & \pm 0.17 & \pm 0.24 & $0.96_{-0.03}^{+0.02}$ \\
\hline$\eta^{\gamma}$ & $\mathrm{d} \sigma / \mathrm{d} \eta^{\gamma}$ & Uncorr. & Corr. & $f_{\text {corr }}$ \\
\hline$[-1.00,-0.57]$ & {$[\mathrm{pb}]$} & \pm 1.3 & \pm 2.5 & $0.99_{-0.04}^{+0.02}$ \\
{$[-0.57,0.20]$} & 18.4 & \pm 1.5 & \pm 1.5 & $0.94_{-0.03}^{+0.02}$ \\
{$[0.20,0.94]$} & 23.9 & \pm 1.2 & \pm 2.3 & $0.90_{-0.04}^{+0.01}$ \\
{$[0.94,1.42]$} & 27.7 & \pm 1.3 & \pm 3.0 & $0.87_{-0.04}^{+0.03}$ \\
{$[1.42,2.40]$} & 19.3 & \pm 1.0 & \pm 3.5 & $0.84_{-0.05}^{+0.02}$ \\
\hline
\end{tabular}

The input is binned in three dimensions in the reconstructed quantities $E_{T}^{\gamma}, \eta^{\gamma}$ and $D$; the latter allows the discrimination of signal and background. The output of the unfolding procedure contains the number of signal events $N_{\text {sig }}$ in $E_{T}^{\gamma}-\eta^{\gamma}$ bins on hadron level and the amount of background events in any of the input bins. Additional underflow and overflow bins are defined for each output variable. Therefore the unfolding matrix $\mathbf{A}$ also includes migrations into or out of the phase space of the measurement. It is computed using signal and background PYTHIA simulation. For measurements including jet-related variables, both the input and the output is additionally binned in some variable $u$, where $u$ is $E_{T}^{\mathrm{jet}}, \eta^{\mathrm{jet}}, x_{\gamma}, x_{p}, \Delta \Phi$ or $p_{\perp}$.

The stability of the unfolding procedure is checked by varying the number of input bins and changing the bin boundaries. The results from the unfolding procedure are compared to a bin-by-bin correction method. Agreement is seen within errors for most of the analysis bins.

Cross sections are presented for $Q^{2}<1 \mathrm{GeV}^{2}$. The extracted number of signal events in each bin $N_{\text {sig }}$ is corrected for a small contribution of DIS events at virtualities $1<Q^{2}<4 \mathrm{GeV}^{2}$. For this kinematic region the scattered electron has a non-negligible probability to escape detection. If such events contain in addition photons at high transverse momentum, their signatures are very similar to the signal process. The corresponding correction factor $f_{\text {DIS }}$ is determined with the PYTHIA signal MC and is found to be above 0.98 for most of the analysis bins. The bin-averaged double differential cross section on hadron level is obtained as

$\frac{\mathrm{d}^{2} \sigma}{\mathrm{d} E_{T}^{\gamma} \mathrm{d} \eta^{\gamma}}=\frac{N_{\text {sig }} \cdot f_{\text {DIS }}}{\left(\mathcal{L} \cdot \Delta E_{T}^{\gamma} \Delta \eta^{\gamma}\right)}$, where $\mathcal{L}$ is the luminosity, $\Delta E_{T}^{\gamma}\left(\Delta \eta^{\gamma}\right)$ is the bin width in $E_{T}^{\gamma}\left(\eta^{\gamma}\right)$ and $N_{\text {sig }}$ corresponds to the number of signal events in the bin $E_{T}^{\gamma}-\eta^{\gamma}$. Single differential cross sections as a function of $E_{T}^{\gamma}\left(\eta^{\gamma}\right)$ are then obtained by summing bins of the double differential cross sections in $\eta^{\gamma}\left(E_{T}^{\gamma}\right)$, taking into account the respective bin widths. The total inclusive cross section is obtained by summing the measured double differential cross section over all analysis bins. The differential cross sections in bins of some jet-related variable $u$ is obtained by unfolding triple-differential cross sections in $E_{T}^{\gamma}, \eta^{\gamma}$ and $u$, which then are summed over the bins in $E_{T}^{\gamma}$ and $\eta^{\gamma}$. For the calculation of cross section uncertainties, correlations between bins are taken into account.

\subsection{Systematic uncertainties}

The following experimental uncertainties are considered:

- The measured shower shape variables in the DVCS and $\mathrm{BH}$ event samples defined in Sect. 4.2 are compared to MC simulations. The uncertainty on the shower shape simulation for the photon is estimated by varying the discriminating variables within the limits deduced from the differences between data and simulation. The uncertainty of the description of the background composition and the shower shapes of neutral hadrons is obtained accordingly by comparing the shower shapes of the BG event sample with the background MC from PYTHIA. The resulting variation of the total inclusive cross section is $11 \%$. The uncertainty varies between $10 \%$ and $25 \%$ for the single differential cross sections increasing towards large $\eta^{\gamma}$.

- An uncertainty of $1 \%$ is attributed to the energy of the photon[46] and an uncertainty of $3 \mathrm{mrad}$ to the measure- 
Fig. 6 Inclusive double differential prompt photon cross sections $\mathrm{d}^{2} \sigma / \mathrm{d} E_{T}^{\gamma} \mathrm{d} \eta^{\gamma}$ for five bins in $\eta^{\gamma}$. The errors between different $\eta^{\gamma}$ bins are correlated. The kinematic range is specified in Table 1. The cross sections are compared to theoretical calculations (see caption of Fig. 5). In addition the prediction from PYTHIA [17] is shown as dashed line

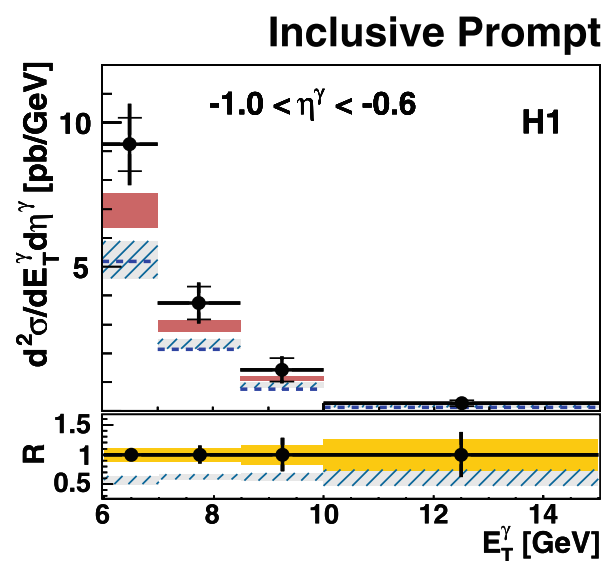

Photon Cross Sections
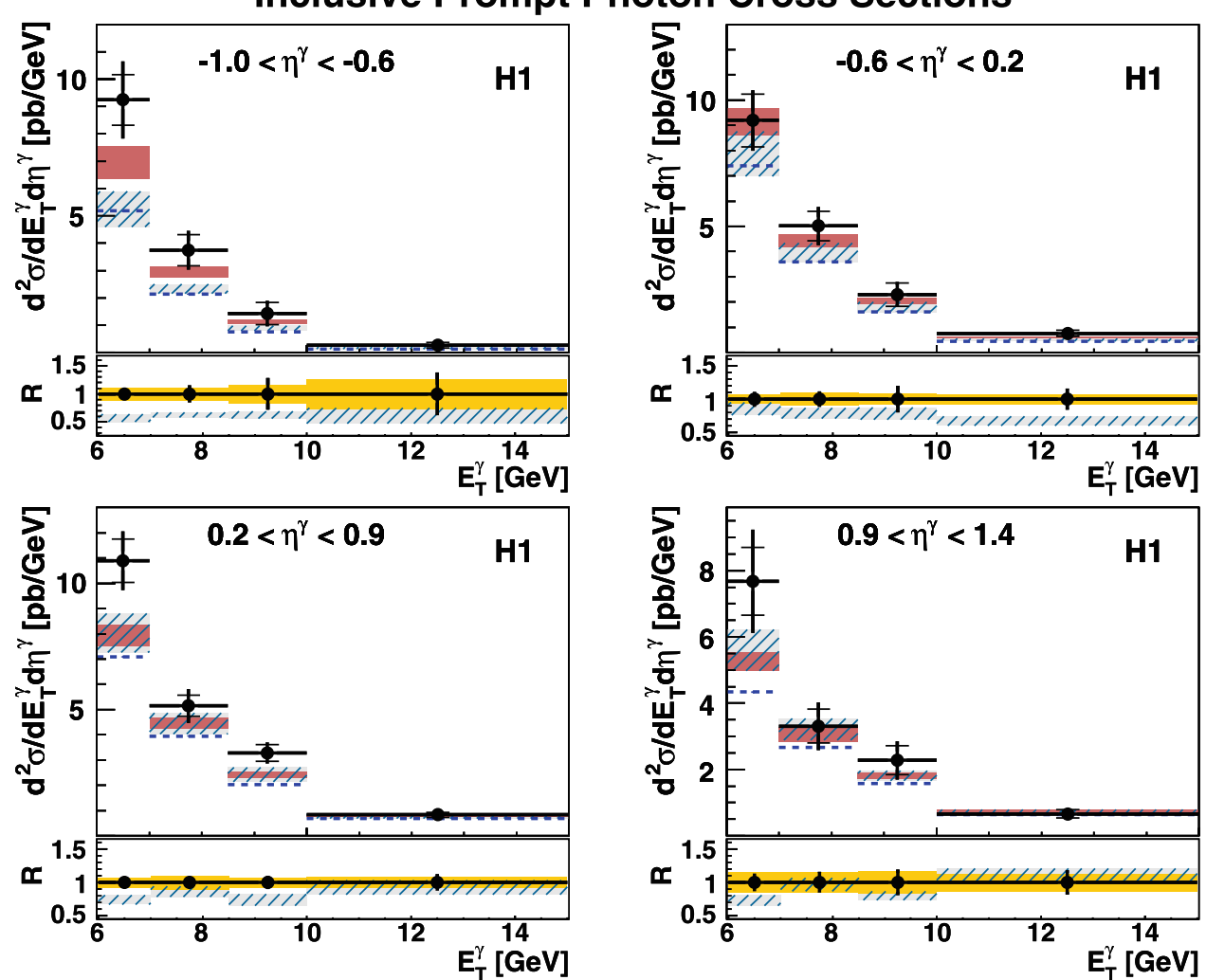

ment of the scattering angle [2] for events with $\eta^{\gamma}<1.4$. For $\eta^{\gamma}>1.4$ the uncertainty is $4 \%$ on the energy scale and $4 \mathrm{mrad}$ on the scattering angle. The resulting error on the total inclusive cross section is $\pm 1.5 \%$.

- A $2 \%$ uncertainty is attributed to the measurement of the hadronic energy [45]. The corresponding uncertainty of the total cross section amounts to $\pm 1 \%$.

- An uncertainty of $\pm 3 \%$ is attributed to the determination of the trigger efficiency.

- The uncertainty on the CIP2k and track veto efficiency results in an error of $\pm 2.5 \%$ on the total inclusive cross section.

- Background from DIS events leads to a systematic uncertainty of $1.5 \%$.
- An uncertainty in the description of the dead material in the simulation is accounted for by varying the probability of photon conversion before the calorimeter by $\pm 10 \%$. For polar angles $\theta<20^{\circ}$ it is varied by $\pm 30 \%$ because of more dead material in the forward region. This results in a $1 \%$ uncertainty for the cross section measurements in the central region and $3 \%$ in the most forward $\eta^{\gamma}$ bin.

- The ratio of resolved to direct photoproduction events in the MC simulation is changed within limits deduced from the measured $x_{\gamma}$ distribution [45], leading to $\pm 1 \%$ systematic error due to a different acceptance.

- The luminosity measurement has an error of $3.4 \%$.

The effects of each systematic error on the cross sections are determined by evaluating an alternative unfolding matrix 
Table 3 Bin averaged double differential cross section for inclusive prompt photon production in bins of transverse energy and pseudorapidity of the photon. The errors are correlated between different $E_{T}^{\gamma}$ and different $\eta^{\gamma}$ bins. More details are given in the caption of Table 2

\begin{tabular}{|c|c|c|c|c|c|}
\hline \multicolumn{6}{|c|}{ H1 inclusive prompt photon cross sections } \\
\hline$\overline{\eta^{\gamma}}$ & $\begin{array}{l}E_{T}^{\gamma} \\
{[\mathrm{GeV}]}\end{array}$ & $\mathrm{d}^{2} \sigma / \mathrm{d} E_{T}^{\gamma} \mathrm{d} \eta^{\gamma}$ & $\begin{array}{l}\text { Uncorr. } \\
{[\mathrm{pb} / \mathrm{GeV}]}\end{array}$ & Corr. & $f_{\text {corr }}$ \\
\hline \multirow[t]{4}{*}[-1.00,-0.57]{} & {$[6.00,7.00]$} & 9.24 & \pm 0.93 & \pm 1.06 & $0.99_{-0.04}^{+0.02}$ \\
\hline & {$[7.00,8.50]$} & 3.75 & \pm 0.57 & \pm 0.44 & $0.99_{-0.05}^{+0.02}$ \\
\hline & {$[8.50,10.00]$} & 1.43 & \pm 0.41 & \pm 0.24 & $0.99_{-0.02}^{+0.02}$ \\
\hline & {$[10.00,15.00]$} & 0.27 & \pm 0.10 & \pm 0.07 & $1.01_{-0.03}^{+0.01}$ \\
\hline \multirow[t]{4}{*}[-0.57,0.20]{} & {$[6.00,7.00]$} & 9.19 & \pm 1.04 & \pm 0.59 & $0.93_{-0.04}^{+0.02}$ \\
\hline & {$[7.00,8.50]$} & 5.02 & \pm 0.59 & \pm 0.48 & $0.94_{-0.02}^{+0.02}$ \\
\hline & {$[8.50,10.00]$} & 2.29 & \pm 0.46 & \pm 0.19 & $0.96_{-0.03}^{+0.02}$ \\
\hline & {$[10.00,15.00]$} & 0.76 & \pm 0.12 & \pm 0.06 & $1.00_{-0.03}^{+0.02}$ \\
\hline \multirow[t]{4}{*}[0.20,0.94]{} & {$[6.00,7.00]$} & 10.90 & \pm 0.86 & \pm 0.78 & $0.87_{-0.05}^{+0.01}$ \\
\hline & {$[7.00,8.50]$} & 5.15 & \pm 0.43 & \pm 0.52 & $0.89_{-0.03}^{+0.02}$ \\
\hline & {$[8.50,10.00]$} & 3.28 & \pm 0.34 & \pm 0.24 & $0.94_{-0.04}^{+0.01}$ \\
\hline & {$[10.00,15.00]$} & 0.83 & \pm 0.10 & \pm 0.07 & $0.96_{-0.04}^{+0.02}$ \\
\hline \multirow[t]{4}{*}[0.94,1.42]{} & {$[6.00,7.00]$} & 7.68 & \pm 1.02 & \pm 1.18 & $0.83_{-0.04}^{+0.04}$ \\
\hline & {$[7.00,8.50]$} & 3.31 & \pm 0.51 & \pm 0.51 & $0.85_{-0.05}^{+0.02}$ \\
\hline & {$[8.50,10.00]$} & 2.27 & \pm 0.44 & \pm 0.38 & $0.90_{-0.04}^{+0.03}$ \\
\hline & {$[10.00,15.00]$} & 0.66 & \pm 0.12 & \pm 0.09 & $0.95_{-0.03}^{+0.03}$ \\
\hline \multirow[t]{4}{*}[1.42,2.43]{} & {$[6.00,7.00]$} & 4.54 & \pm 0.90 & \pm 1.29 & $0.79_{-0.05}^{+0.02}$ \\
\hline & {$[7.00,8.50]$} & 2.12 & \pm 0.41 & \pm 0.72 & $0.82_{-0.05}^{+0.02}$ \\
\hline & {$[8.50,10.00]$} & 0.86 & \pm 0.31 & \pm 0.35 & $0.88_{-0.06}^{+0.02}$ \\
\hline & {$[10.00,15.00]$} & 0.40 & \pm 0.08 & \pm 0.09 & $0.93_{-0.03}^{+0.03}$ \\
\hline
\end{tabular}

$\mathbf{A}^{\prime}$ using the MC prediction made with the corresponding systematic variation applied. The differences to the default unfolding matrix $\mathbf{A}^{\prime}-\mathbf{A}$ are used to evaluate the contributions to the error matrices of the results using standard error propagation. The final error matrix is split into fully correlated and fully uncorrelated parts which are listed in Tables 2 to 7. The systematic uncertainty obtained on the total inclusive cross section is $\pm 13 \%$. The largest contribution to this uncertainty arises from the systematic uncertainties attributed to the description of the shower shapes.

\section{Results}

The prompt photon cross sections presented below are given for the phase space defined in Table 1.

Bin averaged differential cross sections are presented in Figs. 5 to 9 and in Tables 2 to 7 . For all measurements the total uncertainty is dominated by the systematic errors.
The figures also show the ratio of the NLO QCD prediction (FGH) $[6,7]$ to the measured cross section $R=\sigma_{\mathrm{FGH}} / \sigma_{\text {meas }}$ with the uncertainty of the NLO calculation. The factors $f_{\text {corr }}$ (see Sect. 2) for the correction of the theoretical calculations for hadronization, multi-parton interactions and the definition of the isolation are given in the cross section tables with their errors.

The measured inclusive prompt photon cross section in the phase space defined in Table 1 is

$\sigma(e p \rightarrow e \gamma X)=66.9 \pm 1.8$ (stat) \pm 8.7 (syst) pb.

Both calculations predict lower cross sections of $52.1_{-3.4}^{+5.3} \mathrm{pb}$ (FGH) and $56.7_{-3.1}^{+2.3} \mathrm{pb}(\mathrm{LZ})$, while the MC expectation from PYTHIA is $46.4 \mathrm{pb}$. Theoretical uncertainties due to missing higher orders are estimated by simultaneously varying $\mu_{R}$ and $\mu_{F}$ by a factor of 0.5 to 2.0 . In addition, the errors on the theoretical predictions include uncertainties due 


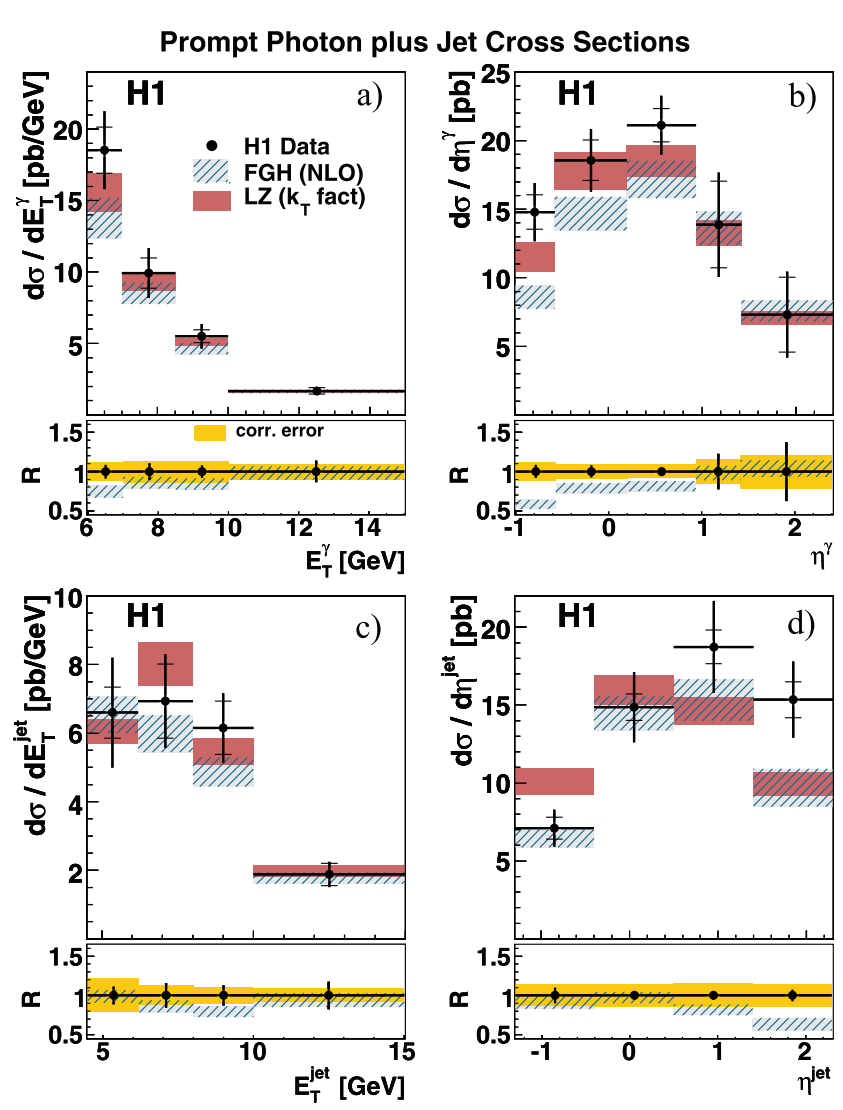

Fig. 7 Differential prompt photon plus jet cross sections (a) $\mathrm{d} \sigma / \mathrm{d} E_{T}^{\gamma}$, (b) $\mathrm{d} \sigma / \mathrm{d} \eta^{\gamma}$, (c) $\mathrm{d} \sigma / \mathrm{d} E_{T}^{\text {jet }}$ and (d) $\mathrm{d} \sigma / \mathrm{d} \eta^{\text {jet }}$ in the kinematic range specified in Table 1. The cross sections are compared to theoretical calculations (see caption of Fig. 5)

to the error of $f_{\text {corr }}$ and due to the PDFs. All these error sources are added in quadrature.

Differential inclusive prompt photon cross sections $\mathrm{d} \sigma / \mathrm{d} E_{T}^{\gamma}$ and $\mathrm{d} \sigma / \mathrm{d} \eta^{\gamma}$ are presented in Table 2 and in Fig. 5. The results are compared to a QCD calculation based on the collinear factorization in NLO (FGH) $[6,7]$, to a calculation based on the $k_{T}$ factorization approach (LZ) [11]. Both calculations are below the data, most significantly at low $E_{T}^{\gamma}$. The $\mathrm{LZ}$ calculation gives a reasonable description of the shape of $\eta^{\gamma}$, whereas the FGH calculation is significantly below the data for central and backward photons $\left(\eta^{\gamma}<0.9\right)$.

Double differential cross sections $d^{2} \sigma / \mathrm{d} E_{T}^{\gamma} \mathrm{d} \eta^{\gamma}$ are shown in Fig. 6 and Table 3 for all five bins in $\eta^{\gamma}$. The $\eta^{\gamma}$ bins correspond to the wheel structure of the LAr calorimeter. LZ provides a reasonable description of the data with the exception of the lowest $E_{T}^{\gamma}$ bin in the central $\eta^{\gamma}(0.2<$ $\left.\eta^{\gamma}<0.9\right)$ region. The FGH calculation underestimates the cross section in the central $\left(0.2<\eta^{\gamma}<0.9\right)$ and backward $\left(\eta^{\gamma}<-0.6\right)$ region. Here, it is significantly below the data. The prediction from PYTHIA is also shown. It underestimates the measured cross section by roughly $45 \%$, most significantly at low $E_{T}^{\gamma}$.
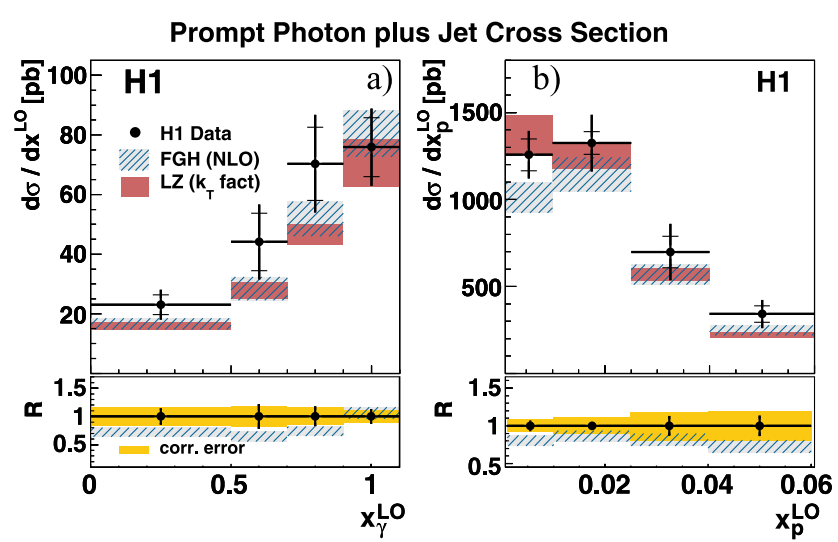

Fig. 8 Differential prompt photon plus jet cross sections (a) $\mathrm{d} \sigma / \mathrm{d} x_{\gamma}^{\mathrm{LO}}$ and (b) $\mathrm{d} \sigma / \mathrm{d} x_{p}^{\mathrm{LO}}$, in the kinematic range specified in Table 1. The cross sections are compared to theoretical calculations (see caption of Fig. 5)

The prompt photon plus jet cross section is

$\sigma(e p \rightarrow e \gamma$ jet $X)=50.1 \pm 1.7$ (stat) \pm 6.5 (syst) pb.

It is similar to the inclusive cross section, since the prompt photon recoils most of the time against a prominent hadronic jet. The theoretical calculations predict cross sections of $40.6_{-1.9}^{+5.3} \mathrm{pb}(\mathrm{FGH})$ and $45.7_{-2.1}^{+4.7} \mathrm{pb}$ (LZ). Both are compatible with the measurement within the errors. The PYTHIA expectation of $33.9 \mathrm{pb}$ is again too low.

Cross sections for the production of a prompt photon plus jet are presented in Fig. 7 and Tables 4, 5 as a function of the variables $E_{T}^{\gamma}, \eta^{\gamma}, E_{T}^{\text {jet }}$ and $\eta^{\text {jet }}$. Both calculations give a reasonable description of the $E_{T}^{\gamma}$ and $E_{T}^{\mathrm{jet}}$ cross sections but show deficits in the description of the $\eta^{\text {jet }}$ shape. Here, the LZ prediction is too high for jets with $\eta^{\text {jet }}<0.5$, and both calculations underestimate the rate of events with forward jets. As in the inclusive case, the FGH prediction is too low for $\eta^{\gamma}<0.2$.

Photon plus jet cross section as a function of the estimators $x_{\gamma}^{\mathrm{LO}}$ and $x_{p}^{\mathrm{LO}}$ are shown in Fig. 8 and Table 4. Both distributions are described by the calculations within errors.

Cross sections for the two observables describing the transverse correlation between the photon and the jet, $p_{\perp}$ and $\Delta \Phi$, are shown in Fig. 9 and Tables 6, 7. Both variables are expected to be sensitive to higher order gluon emission. The phase-space is divided into two parts: one with $x_{\gamma}^{\mathrm{LO}}>0.8$ where the direct interaction of a photon with the proton dominates and one with $x_{\gamma}^{\mathrm{LO}}<0.8$, including significant contributions from events with a resolved photon. For $x_{\gamma}^{\mathrm{LO}}>0.8$ both predictions underestimate the tails of the distributions suggesting that there is more decorrelation in the data than predicted. For $x_{\gamma}^{\mathrm{LO}}<0.8$ the $p_{\perp}$ distribution is harder than for $x_{\gamma}^{\mathrm{LO}}>0.8$, which reflects the increased contributions from events with a resolved photon and from photons radiated from quarks in di-jet events. The 
Table 4 Bin averaged differential cross section for prompt photon plus jet production as a function of $E_{T}^{\gamma}$, $\eta^{\gamma}, E_{T}^{\mathrm{jet}}$ and $\eta^{\mathrm{jet}}$. More details are given in the caption of Table 2

\begin{tabular}{|c|c|c|c|c|}
\hline \multicolumn{5}{|c|}{ H1 prompt photon plus jet cross sections } \\
\hline $\begin{array}{l}E_{T}^{\gamma} \\
{[\mathrm{GeV}]}\end{array}$ & $\mathrm{d} \sigma / \mathrm{d} E_{T}^{\gamma}$ & $\begin{array}{l}\text { Uncorr. } \\
{[\mathrm{pb} / \mathrm{GeV}]}\end{array}$ & Corr. & $f_{\text {corr }}$ \\
\hline$[6.0,7.0]$ & 18.53 & \pm 1.61 & \pm 2.21 & $0.82_{-0.06}^{+0.05}$ \\
\hline$[7.0,8.5]$ & 9.93 & \pm 1.06 & \pm 1.39 & $0.87_{-0.05}^{+0.04}$ \\
\hline$[8.5,10.0]$ & 5.50 & \pm 0.45 & \pm 0.73 & $0.92_{-0.04}^{+0.03}$ \\
\hline$[10.0,15.0]$ & 1.68 & \pm 0.23 & \pm 0.16 & $0.95_{-0.03}^{+0.03}$ \\
\hline$\eta^{\gamma}$ & $\mathrm{d} \sigma / \mathrm{d} \eta^{\gamma}$ & $\begin{array}{l}\text { Uncorr. } \\
{[\mathrm{pb}]}\end{array}$ & Corr. & $f_{\text {corr }}$ \\
\hline$[-1.00,-0.57]$ & 14.79 & \pm 1.25 & \pm 1.70 & $0.94_{-0.07}^{+0.06}$ \\
\hline$[-0.57,0.20]$ & 18.57 & \pm 1.47 & \pm 1.75 & $0.90_{-0.05}^{+0.04}$ \\
\hline$[0.20,0.94]$ & 21.12 & \pm 1.21 & \pm 1.77 & $0.87_{-0.04}^{+0.03}$ \\
\hline$[0.94,1.42]$ & 13.88 & \pm 3.15 & \pm 2.12 & $0.86_{-0.04}^{+0.04}$ \\
\hline$[1.42,2.40]$ & 7.31 & \pm 2.73 & \pm 1.54 & $0.84_{-0.05}^{+0.03}$ \\
\hline $\begin{array}{l}E_{T}^{\mathrm{jet}} \\
{[\mathrm{GeV}]}\end{array}$ & $\mathrm{d} \sigma / \mathrm{d} E_{T}^{\mathrm{jet}}$ & $\begin{array}{r}\text { Uncorr. } \\
{[\mathrm{pb} / \mathrm{GeV}]}\end{array}$ & Corr. & $f_{\text {corr }}$ \\
\hline$[4.5,6.2]$ & 6.60 & \pm 0.74 & \pm 1.42 & $0.85_{-0.05}^{+0.04}$ \\
\hline$[6.2,8.0]$ & 6.93 & \pm 1.08 & \pm 0.84 & $0.83_{-0.06}^{+0.05}$ \\
\hline$[8.0,10.0]$ & 6.15 & \pm 0.78 & \pm 0.65 & $0.90_{-0.05}^{+0.03}$ \\
\hline$[10.0,15.0]$ & 1.88 & \pm 0.33 & \pm 0.17 & $0.96_{-0.04}^{+0.03}$ \\
\hline$\eta^{\text {jet }}$ & $\mathrm{d} \sigma / \mathrm{d} \eta^{\mathrm{jet}}$ & $\begin{array}{l}\text { Uncorr. } \\
{[\mathrm{pb}]}\end{array}$ & Corr. & $f_{\text {corr }}$ \\
\hline$[-1.3,-0.4]$ & 7.1 & \pm 0.7 & \pm 1.0 & $0.80_{-0.04}^{+0.03}$ \\
\hline$[-0.4,0.5]$ & 14.9 & \pm 0.8 & \pm 2.1 & $0.86_{-0.04}^{+0.03}$ \\
\hline$[0.5,1.4]$ & 18.7 & \pm 1.1 & \pm 2.7 & $0.91_{-0.06}^{+0.04}$ \\
\hline$[1.4,2.3]$ & 15.3 & \pm 1.2 & \pm 2.2 & $0.94_{-0.06}^{+0.04}$ \\
\hline
\end{tabular}

FGH calculation poorly describes the $p_{\perp}$ distribution but gives a reasonable description of the measurement in $\Delta \Phi$ for $x_{\gamma}^{\mathrm{LO}}<0.8$, except for the highest bin in $\Delta \Phi$. The regions $\Delta \Phi \rightarrow 180^{\circ}$ and $p_{\perp} \rightarrow 0$ are sensitive to multiple soft gluon radiation which limits the validity of fixed order calculations [47]. The LZ calculation includes multiple gluon radiation in the initial state before the hard subprocess and describes $\Delta \Phi>170^{\circ}$ and $p_{\perp}<2 \mathrm{GeV}$, but predicts a significantly lower contribution of events in the tails of both distributions as compared to the data.

The present measurement is compared to the published results of $\mathrm{H1}$ [1] and ZEUS [5] in the restricted phase space $0.2<y_{h}<0.7$. For the comparison with the inclusive measurement of $\mathrm{H} 1$ the $\eta^{\gamma}$ range is restricted to $-1.0<\eta^{\gamma}<$ 0.9. For the comparison with the ZEUS results for isolated photons with a jet, the kinematic range is changed to $7<$
$E_{T}^{\gamma}<15 \mathrm{GeV}, 6<E_{T}^{\mathrm{jet}}<17 \mathrm{GeV}$ and $-1.6<\eta^{\text {jet }}<2.4$. The results of this analysis are found in agreement with the previous measurements [45].

\section{Conclusions}

The photoproduction of prompt photons is measured in $e p$ collisions at a center-of-mass energy of $319 \mathrm{GeV}$ with the H1 detector at HERA using a data sample corresponding to an integrated luminosity of $340 \mathrm{pb}^{-1}$. Photons with a transverse energy in the range $6<E_{T}^{\gamma}<15 \mathrm{GeV}$ and with pseudorapidity $-1.0<\eta^{\gamma}<2.4$ are measured in the kinematic region $Q^{2}<1 \mathrm{GeV}^{2}$ and $0.1<y<0.7$. Compared to previous measurements, the range of $\eta^{\gamma}$ is significantly extended, and the luminosity of the measurement is increased by a factor three. 
Table 5 Bin averaged differential cross section for prompt photon plus jet production as a function of $x_{\gamma}^{\mathrm{LO}}$ and $x_{p}^{\mathrm{LO}}$. More details are given in the caption of Table 2

Table 6 Bin averaged differential cross sections for prompt photon plus jet production as a function of $p_{\perp}$, the photon's momentum perpendicular to the jet direction in the transverse plane, separated into two regions with $x_{\gamma}^{\mathrm{LO}}>0.8$ and $x_{\gamma}^{\mathrm{LO}}<0.8$. More details are given in the caption of Table 2

Table 7 Bin averaged differential cross sections for prompt photon plus jet production as a function of $\Delta \Phi$, the difference in azimuthal angle between the photon and the jet, separated into two regions with $x_{\gamma}^{\mathrm{LO}}>0.8$ and $x_{\gamma}^{\mathrm{LO}}<0.8$. More details are given in the caption of Table 2

\begin{tabular}{|c|c|c|c|c|}
\hline \multicolumn{5}{|c|}{ H1 prompt photon plus jet cross sections } \\
\hline$x_{\gamma}^{\mathrm{LO}}$ & $\mathrm{d} \sigma / \mathrm{d} x_{\gamma}^{\mathrm{LO}}$ & $\begin{array}{l}\text { Uncorr. } \\
{[\mathrm{pb}]}\end{array}$ & Corr. & $f_{\text {corr }}$ \\
\hline$[0.0,0.5]$ & 23.0 & \pm 3.3 & \pm 3.8 & $0.78_{-0.08}^{+0.04}$ \\
\hline$[0.5,0.7]$ & 44.1 & \pm 9.6 & \pm 8.1 & $0.89_{-0.08}^{+0.06}$ \\
\hline$[0.7,0.9]$ & 70.3 & \pm 12.3 & \pm 10.9 & $1.24_{-0.06}^{+0.04}$ \\
\hline$[0.9,1.1]$ & 75.9 & \pm 9.8 & \pm 8.4 & $0.82_{-0.04}^{+0.04}$ \\
\hline$x_{p}^{\mathrm{LO}}$ & $\mathrm{d} \sigma / \mathrm{d} x_{p}^{\mathrm{LO}}$ & $\begin{array}{l}\text { Uncorr. } \\
{[\mathrm{pb}]}\end{array}$ & Corr. & $f_{\text {corr }}$ \\
\hline$[0.001,0.010]$ & 1257 & \pm 91 & \pm 102 & $0.84_{-0.05}^{+0.04}$ \\
\hline$[0.010,0.025]$ & 1325 & \pm 65 & \pm 150 & $0.88_{-0.05}^{+0.04}$ \\
\hline$[0.025,0.040]$ & 698 & \pm 90 & \pm 134 & $0.90_{-0.05}^{+0.03}$ \\
\hline$[0.040,0.060]$ & 341 & \pm 47 & \pm 66 & $0.91_{-0.06}^{+0.03}$ \\
\hline
\end{tabular}

\begin{tabular}{|c|c|c|c|c|c|}
\hline \multicolumn{6}{|c|}{ H1 prompt photon plus jet cross sections } \\
\hline$\overline{x_{\gamma}^{\mathrm{LO}}}$ & $\begin{array}{l}p_{\perp} \\
{[\mathrm{GeV}]}\end{array}$ & $\mathrm{d} \sigma / \mathrm{d} p_{\perp}$ & $\begin{array}{l}\text { Uncorr. } \\
{[\mathrm{pb} / \mathrm{GeV}]}\end{array}$ & Corr. & $f_{\text {corr }}$ \\
\hline \multirow[t]{4}{*}[0.8,1.1]{} & {$[0,2]$} & 7.75 & \pm 0.50 & \pm 0.32 & $0.89_{-0.04}^{+0.04}$ \\
\hline & {$[2,4]$} & 3.96 & \pm 0.43 & \pm 0.53 & $0.87_{-0.01}^{+0.01}$ \\
\hline & {$[4,6]$} & 2.16 & \pm 0.55 & \pm 0.56 & $0.84_{-0.07}^{+0.07}$ \\
\hline & {$[6,8]$} & 0.60 & \pm 0.53 & \pm 0.36 & $0.81_{-0.08}^{+0.08}$ \\
\hline \multirow[t]{4}{*}[0.0,0.8]{} & {$[0,2]$} & 7.14 & \pm 0.48 & \pm 1.04 & $0.84_{-0.11}^{+0.08}$ \\
\hline & {$[2,4]$} & 4.52 & \pm 0.49 & \pm 0.80 & $0.91_{-0.08}^{+0.03}$ \\
\hline & {$[4,6]$} & 2.91 & \pm 0.40 & \pm 0.56 & $0.96_{-0.04}^{+0.03}$ \\
\hline & {$[6,8]$} & 2.35 & \pm 0.44 & \pm 0.37 & $1.07_{-0.10}^{+0.09}$ \\
\hline
\end{tabular}

\begin{tabular}{|c|c|c|c|c|c|}
\hline \multicolumn{6}{|c|}{ H1 prompt photon plus jet cross sections } \\
\hline$\overline{x_{\gamma}^{\mathrm{LO}}}$ & $\Delta \Phi$ & $\mathrm{d} \sigma / \mathrm{d} \Delta \Phi$ & $\begin{array}{l}\text { Uncorr. } \\
{[\mathrm{pb}]}\end{array}$ & Corr. & $f_{\text {corr }}$ \\
\hline \multirow[t]{4}{*}[0.8,1.1]{} & {$[130,150]$} & 0.19 & \pm 0.04 & \pm 0.01 & $0.82_{-0.04}^{+0.03}$ \\
\hline & {$[150,165]$} & 0.46 & \pm 0.06 & \pm 0.06 & $0.84_{-0.01}^{+0.01}$ \\
\hline & {$[165,172]$} & 0.89 & \pm 0.14 & \pm 0.07 & $0.93_{-0.01}^{+0.00}$ \\
\hline & {$[172,180]$} & 1.38 & \pm 0.04 & \pm 0.17 & $0.89_{-0.05}^{+0.05}$ \\
\hline \multirow[t]{4}{*}[0.0,0.8]{} & {$[130,150]$} & 0.27 & \pm 0.03 & \pm 0.04 & $0.94_{-0.05}^{+0.03}$ \\
\hline & {$[150,165]$} & 0.52 & \pm 0.06 & \pm 0.08 & $0.91_{-0.08}^{+0.04}$ \\
\hline & {$[165,172]$} & 0.91 & \pm 0.14 & \pm 0.18 & $0.83_{-0.09}^{+0.07}$ \\
\hline & {$[172,180]$} & 1.21 & \pm 0.11 & \pm 0.15 & $0.84_{-0.12}^{+0.08}$ \\
\hline
\end{tabular}



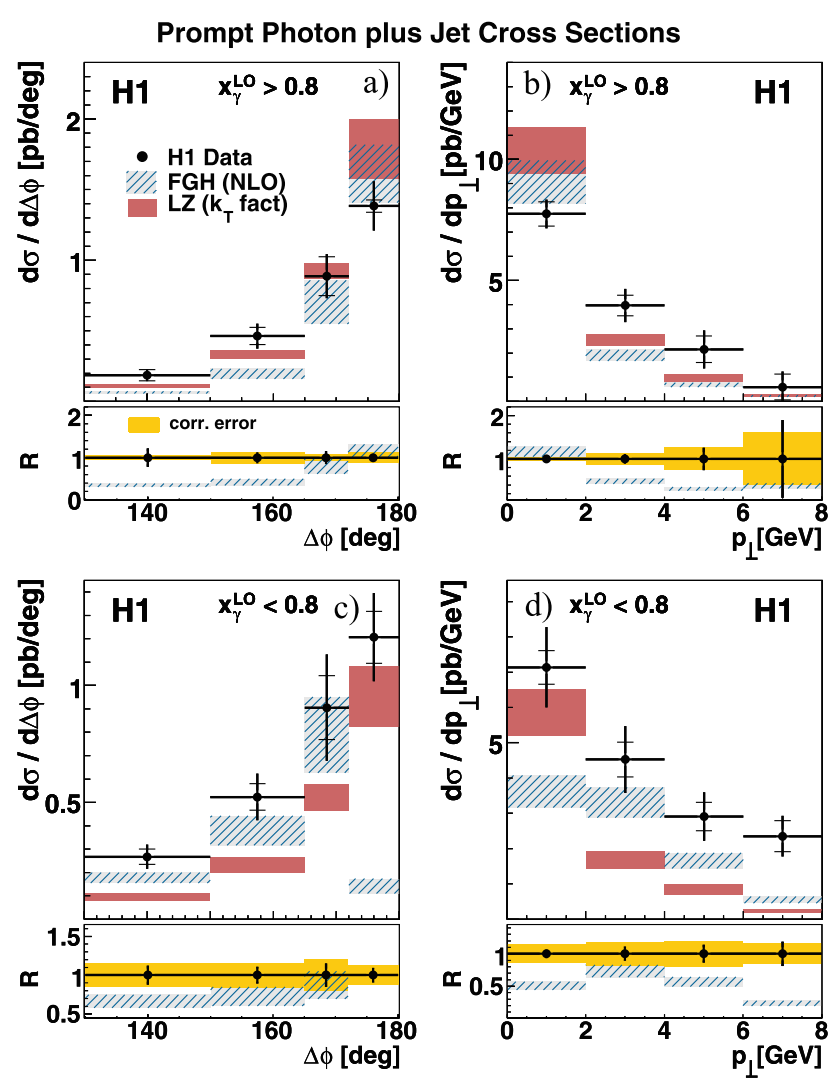

Fig. 9 Differential prompt photon plus jet cross sections $\mathrm{d} \sigma / \mathrm{d} \Delta \Phi$ and $\mathrm{d} \sigma / \mathrm{d} p_{\perp}$ the photon momentum transverse to the jet direction, the difference in azimuthal angle between the photon and the jet. The kinematic range is specified in Table 1. Figures (a) and (b) show the cross section for $x_{\gamma}^{\mathrm{LO}}>0.8,(\mathbf{c})$ and (d) for $x_{\gamma}^{\mathrm{LO}}<0.8$. The cross sections are compared to theoretical calculations (see caption of Fig. 5)

Single differential and double differential cross sections are measured. The data are compared to a QCD calculation based on the collinear factorization in NLO (FGH) $[6,7]$, to a QCD calculation based on the $k_{T}$ factorization approach (LZ) [11], and to the MC prediction from PYTHIA. The predicted total cross section is lower than the measurement by around $20 \%$. Both theoretical calculations underestimate the data at low $E_{T}^{\gamma}$. While the $\mathrm{LZ}$ prediction describes the shape of $\mathrm{d} \sigma / \mathrm{d} \eta^{\gamma}$ reasonably well, the FGH prediction is significantly below the data for backward photons $\left(\eta^{\gamma}<-0.6\right)$. PYTHIA underestimates the data by roughly $45 \%$, most significantly at low $E_{T}^{\gamma}$.

Differential cross sections for photon plus jet are measured as a function of the observables $E_{T}^{\gamma}, \eta^{\gamma}, \eta^{\mathrm{jet}}, E_{T}^{\mathrm{jet}}$, $x_{\gamma}^{\mathrm{LO}}$, and $x_{p}^{\mathrm{LO}}$. The measured cross sections as a function of the transverse energy of the photon and the jet as well as $x_{\gamma}^{\mathrm{LO}}$ and $x_{p}^{\mathrm{LO}}$ are described within errors by the calculations. However, neither of the predictions is able to describe the measured shape as a function of $\eta^{\text {jet }}$.

Correlations in the transverse plane between the jet and the photon are investigated by measurements of the difference in azimuthal angle $\Delta \Phi$ and of the photon's momentum perpendicular to the jet direction, $p_{\perp}$. A significant fraction of events shows a topology which is not back-to-back. Neither calculation is able to describe the measured correlations in the transverse plane.

Prompt photon cross section in photoproduction are now measured at a precision of about $10 \%$, with hadronization corrections known at the level of 5\%. The challenge remains to further improve the theoretical calculations and arrive at a deeper understanding of the underlying QCD dynamics in this interesting channel.

Acknowledgements We are grateful to the HERA machine group whose outstanding efforts have made this experiment possible. We thank the engineers and technicians for their work in constructing and maintaining the $\mathrm{H} 1$ detector, our funding agencies for financial support, the DESY technical staff for continual assistance and the DESY directorate for support and for the hospitality which they extend to the non DESY members of the collaboration. We would like to thank Artem Lipatov and Nikolai Zotov for providing the LZ calculations and Gudrun Heinrich for help with the FGH calculations.

\section{Appendix: Unfolding procedure}

The photon signal is extracted using an unfolding procedure to relate distributions $\vec{y}_{\text {rec }}$ of reconstructed variables to distributions $\vec{x}_{\text {true }}$ of true variables on hadron level, to determine the fractions of signal and background and to correct the data for the detector efficiency. The unfolding matrix $\mathbf{A}$ which reflects the acceptance of the H1 detector relates the two vectors, $\mathbf{A} \vec{x}_{\text {true }}=\vec{y}_{\text {rec }}$. Each matrix element $A_{i j}$ is the probability for an event originating from bin $j$ of $\vec{x}_{\text {true }}$ to be measured in bin $i$ of $\vec{y}_{\text {rec }}$. The matrix $\mathbf{A}$ is computed using the PYTHIA simulation for the signal and the background, interfaced to the GEANT simulation of the $\mathrm{H} 1$ detector.

A schematic view of the simplified unfolding matrix $\mathbf{A}$ is shown in Fig. 10. Each row of the matrix corresponds to one element of the vector $\vec{x}_{\text {true }}$. The elements of $\vec{x}_{\text {true }}$ are: signal, migration and background bins. Each column of the matrix corresponds to one element of the vector $\vec{y}_{\text {rec }}$. The elements of $\vec{y}_{\text {rec }}$ are: reconstructed bins and side bins. When solving the equation for $\vec{x}_{\text {true }}$ the number of efficiency corrected signal, migration and background events is determined in one step.

The input $\vec{y}_{\text {rec }}$ is binned in three dimensions in the reconstructed quantities $E_{T}^{1,2,3}, \eta^{1,2}$ and $D$. The binning in $D$ is required for the discrimination of signal and background. Figure 10 shows $3 \times 2 \times 5$ "Reconstructed Bins". The signal is binned in the hadron-level quantities $E_{T}^{A, B}$ and $\eta^{A, B}$. Figure 10 shows $2 \times 2$ "Signal" bins in these variables.

In addition, $\vec{x}_{\text {true }}$ includes "background" bins in $E_{T}^{1,2,3}$ and $\eta^{1,2}$, in parallel to the reconstructed quantities. These bins give the amount of background in each reconstructed bin. The background is determined in the unfolding together with the signal contribution. 
Fig. 10 Schematic sketch of the unfolding matrix for $2 \times 2$ signal bins and $3 \times 2 \times 5$ reconstructed bins. Two generator cuts $(z$ and $y)$ and the background are taken into account. The size of the boxes reflects the number of entries in a bin

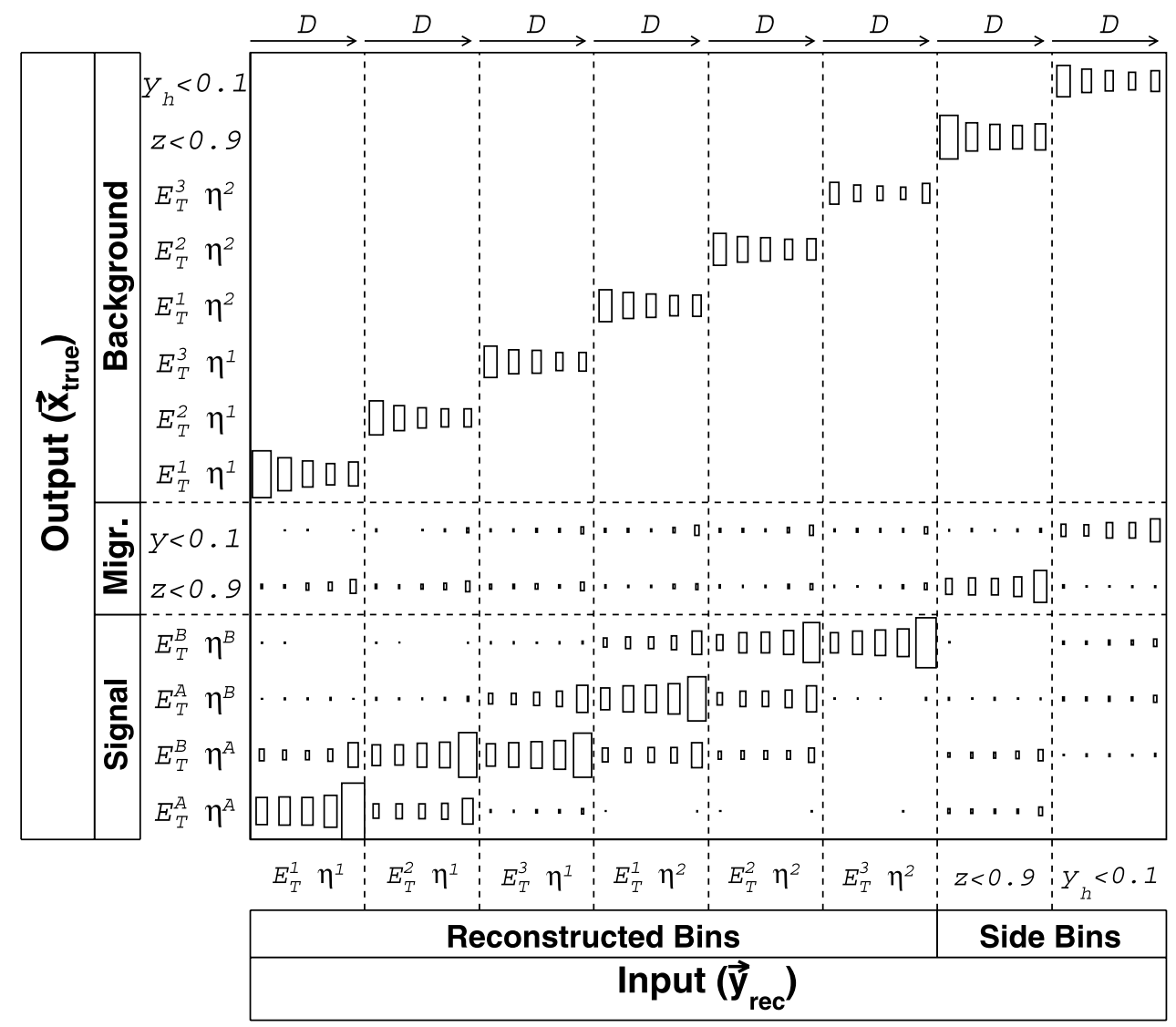

The final unfolding matrix $\mathbf{A}$ also takes into account migrations into or out of the phase space of the measurement. For each cut on hadron level, used to define the measurement phase space (Table 1), a migration bin is added, containing events generated outside of the phase space but reconstructed in any of the input bins. In Fig. 10, two such "Migr." bins are shown. In order to minimize possible biases introduced by the signal MC simulation outside the phase space, each migration bin is subdivided into $E_{T}^{\gamma}$ and $\eta^{\gamma}$ bins (not shown in the figure).

The amount of migration from outside of the generated phase space is controlled by including "Side" bins on detector level for each of the "Migration" bins on hadron level. A side bin is defined as a narrow slice outside the nominal cut value of the reconstructed variable. The side bins are also subdivided into $E_{T}^{\gamma}$ and $\eta^{\gamma}$ bins.

Using matrix A the unfolded distribution $\vec{x}_{\text {true }}$ is obtained from the observed distribution $\vec{y}_{\text {rec }}$ by minimizing a $\chi^{2}$ function given by

$\chi^{2}=\chi_{A}^{2}+\tau^{2} \chi_{L}^{2}$

where

$\chi_{A}^{2}=1 / 2 \cdot\left(\vec{y}_{\text {rec }}-\mathbf{A} \vec{x}_{\text {true }}\right)^{T} \mathbf{V}^{-1}\left(\vec{y}_{\text {rec }}-\mathbf{A} \vec{x}_{\text {true }}\right)$ measures the deviation of $\mathbf{A} \vec{x}_{\text {true }}$ from the data bins $\vec{y}_{\text {rec }}$. Here, $\mathbf{V}=\operatorname{Cov}\left(y_{i}, y_{j}\right)$ is the covariance matrix of the data, initially approximated by the observed statistical errors. In order to avoid a known bias of this procedure [48], the unfolding is iterated using an updated covariance matrix [45], constructed from the expected statistical uncertainties. For a given regularization parameter $\tau$ the regularization term is defined as $\chi_{L}^{2}=\left(\vec{x}_{\text {true }}\right)^{2}$. The minimum $\chi^{2}$ can be calculated analytically and is found as

$\vec{x}_{\text {true }}=\left(\left(\mathbf{A}^{T} \mathbf{V}^{-1} \mathbf{A}\right)+\mathbf{1} \tau\right)^{-1} \mathbf{A}^{T} \mathbf{V}^{-1} \vec{y}_{\text {rec }}$.

The size of the regularization parameter $\tau$ is chosen using the $L$-curve method [49-51].

\section{References}

1. A. Aktas et al. (H1 Collaboration), Eur. Phys. J. C 38, 437 (2005). hep-ex/0407018

2. F.D. Aaron et al. (H1 Collaboration), Eur. Phys. J. C 54, 371 (2008). arXiv:0711.4578

3. J. Breitweg et al. (ZEUS Collaboration), Phys. Lett. B 472(1-2), 175 (2000). hep-ex/9910045

4. S. Chekanov et al. (ZEUS Collaboration), Phys. Lett. B 511, 19 (2001). hep-ex/0104001

5. S. Chekanov et al. (ZEUS Collaboration), Eur. Phys. J. C 49, 511 (2007). hep-ex/0608028 
6. M. Fontannaz, J.P. Guillet, G. Heinrich, Eur. Phys. J. C 21, 303 (2001). hep-ph/0105121

7. M. Fontannaz, G. Heinrich, Eur. Phys. J. C 34, 191 (2004). hep-ph/0312009

8. A. Zembrzuski, M. Krawczyk, hep-ph/0309308

9. A. Gehrmann-De Ridder, T. Gehrmann, E. Poulsen, Phys. Rev. Lett. 96, 132002 (2006). hep-ph/0601073

10. A. Gehrmann-De Ridder, G. Kramer, H. Spiesberger, Nucl. Phys. B 578, 326 (2000). hep-ph/0003082

11. A.V. Lipatov, N.P. Zotov, Phys. Rev. D 72, 054002 (2005). hep-ph/0506044

12. J. Pumplin et al., J. High Energy Phys. 0207, 012 (2002). hep-ph/0201195

13. P. Aurenche, M. Fontannaz, J.P. Guillet, Eur. Phys. J. C 44, 395 (2005). hep-ph/0503259

14. M.A. Kimber, A.D. Martin, M.G. Ryskin, Phys. Rev. D 63, 114027 (2001). hep-ph/0101348

15. M. Glück, E. Reya, A. Vogt, Phys. Rev. D 46, 1973 (1992)

16. M. Glück, E. Reya, A. Vogt, Z. Phys. C 67, 433 (1995)

17. T. Sjöstrand et al., PYTHIA 6.2 Physics and Manual. hep-ph/ 0108264

18. G. Corcella et al., HERWIG 6.5 Release Note 135, 128 (2001). hep-ph/0210213, Version 6.505 is used

19. J.M. Butterworth, J.R. Forshaw, M.H. Seymour, Z. Phys. C 72, 637 (1996). hep-ph/9601371

20. T. Sjöstrand, M. van Zijl, Phys. Rev. D 36, 2019 (1987)

21. M. Bengtsson, T. Sjöstrand, Z. Phys. C 37, 465 (1988)

22. B. Andersson et al., Phys. Rep. 97, 31 (1983)

23. G.A. Schuler, T. Sjöstrand, Phys. Lett. B 376, 193 (1996). hep-ph/9601282

24. H.L. Lai et al. (CTEQ Collaboration), Eur. Phys. J. C 12, 375 (2000). hep-ph/9903282

25. A.D. Martin et al., Phys. Lett. B 604, 61 (2004). hep-ph/0410230

26. R. Brun et al., GEANT 3, CERN_DD/EE/84-1

27. I. Abt et al. (H1 Collaboration), Nucl. Instrum. Methods A 386, 310 (1997)

28. I. Abt et al. (H1 Collaboration), Nucl. Instrum. Methods A 386, 348 (1997)

29. D. Pitzl et al., Nucl. Instrum. Methods A 454, 334 (2000). hep-ex/0002044

30. J. Becker et al., Nucl. Instrum. Methods A 586, 190 (2008). physics $/ 0701002$

31. B. Andrieu et al. (H1 Calorimeter Group), Nucl. Instrum. Methods A 336, 460 (1993)
32. B. Andrieu et al. (H1 Calorimeter Group), Nucl. Instrum. Methods A 350, 57 (1994)

33. B. Andrieu et al. (H1 Calorimeter Group), Nucl. Instrum. Methods A 336, 499 (1993)

34. R.D. Appuhn et al., Nucl. Instrum. Methods A 386, 397 (1997)

35. C. Adloff et al. (H1 Collaboration), Eur. Phys. J. C 30, 1 (2003). hep-ex/0304003

36. S.D. Ellis, D.E. Soper, Phys. Rev. D 48, 3160 (1993). hep-ph/ 9305266

37. M. Fontannaz, J.P. Guillet, G. Heinrich, Eur. Phys. J. C 22, 303 (2001). hep-ph/0107262

38. P. Aurenche, J.P. Guillet, M. Fontannaz, Z. Phys. C 64, 621 (1994). hep-ph/9406382

39. C. Schmitz, Isolated photon production in deep-inelastic scattering at HERA. Ph.D. thesis, Zürich University (2007). Available at http://www-h1.desy.de/publications/theses_list.html

40. P. Domingos, M. Pazzani, Mach. Learn. 29, 103 (1997)

41. D.L. Phillips, J. Assoc. Comput. Mach. 9, 84 (1962)

42. A.N. Tikhanov, Soviet Math. Dokl. 4, 1035 (1963); English translation of Dokl. Akad. Nauk. SSSR 151, 501 (1963)

43. V. Blobel, in Proc. Advanced Statistical Techniques in Particle Physics, Durham (2002)

44. R. Brun, F. Rademakers, Nucl. Instrum. Methods A 389, 81 (1997). ROOT version 5.18 with TUnfold version 14, the latter available at http://www.desy.de/ sschmitt/

45. K. Nowak, Prompt photon production in photoproduction at HERA. Ph.D. thesis, Zürich University, 2009, in preparation, to be made available at http://www-h1.desy.de/publications/ theses_list.html

46. A. Aktas et al. (H1 Collaboration), DESY-09-109. arXiv: 0907.5289

47. H. Gudrun, Private communication

48. G. D’Agostini, Nucl. Instrum. Methods Res. 346, 306 (1994)

49. C.L. Lawson, P.C. Hansen, Solving Least Squares Problems (Prentice-Hall, Englewood Cliffs, 1974); reprinted by SIAM, Philadelphia, 1995

50. K. Miller, SIAM J. Math. Anal. 1, 52 (1970)

51. P.C. Hansen, The L-curve and its use in the numerical treatment of inverse problems, in Computational Inverse Problems in Electrocardiology, Advances in Computational Bioengineering, ed. by P. Johnston (WIT Press, Southampton, 2000), pp. 119-142 